\title{
A Wireless Sensor Network for RF-Based Indoor Localization
}

\author{
Ville A. Kaseva, ${ }^{1}$ Mikko Kohvakka, ${ }^{1}$ Mauri Kuorilehto, ${ }^{2}$ Marko Hännikäinen, ${ }^{1}$ and Timo D. Hämäläinen ${ }^{1}$ \\ ${ }^{1}$ Department of Computer Systems, Tampere University of Technology, P.O. Box 553, 33101 Tampere, Finland \\ ${ }^{2}$ Nokia, Devices R\&D, Visiokatu 3, 33720 Tampere, Finland
}

Correspondence should be addressed to Ville A. Kaseva, ville.a.kaseva@tut.fi

Received 14 August 2007; Revised 11 January 2008; Accepted 26 March 2008

Recommended by Davide Dardari

\begin{abstract}
An RF-based indoor localization design targeted for wireless sensor networks (WSNs) is presented. The energy-efficiency of mobile location nodes is maximized by a localization medium access control (LocMAC) protocol. For location estimation, a location resolver algorithm is introduced. It enables localization with very scarce energy and processing resources, and the utilization of simple and low-cost radio transceiver HardWare (HW) without received signal strength indicator (RSSI) support. For achieving high energy-efficiency and minimizing resource usage, LocMAC is tightly cross-layer designed with the location resolver algorithm. The presented solution is fully calibration-free and can cope with coarse grained and unreliable ranging measurements. We analyze LocMAC power consumption and show that it outperforms current state-of-the-art WSN medium access control (MAC) protocols in location node energy-efficiency. The feasibility of the proposed localization scheme is validated by experimental measurements using real resource constrained WSN node prototypes. The prototype network reaches accuracies ranging from $1 \mathrm{~m}$ to $7 \mathrm{~m}$. With one anchor node per a typical office room, the current room of the localized node is determined with $89.7 \%$ precision.
\end{abstract}

Copyright (C) 2008 Ville A. Kaseva et al. This is an open access article distributed under the Creative Commons Attribution License, which permits unrestricted use, distribution, and reproduction in any medium, provided the original work is properly cited.

\section{INTRODUCTION}

The problem of localization includes determining where a given node is physically located [1]. The existence of location information enables a myriad of functions. At application level, activities such as location and asset tracking, monitoring, context aware applications [2], and personal positioning [3] are made possible. Enabled protocol-level functions include location-based routing $[4,5]$, and geographic addressing [6].

The Global Positioning System (GPS) [7] is a commonly used technology for localization. However, GPS performs poorly in indoor environments. Localization using wireless local area networks (WLANs) has been widely studied as a potential solution for feasible indoor localization [8-16]. However, the application space of both GPS and WLAN localization is limited due to practical considerations such as the size, form factor, cost, and relatively large power consumption of the nodes.

Wireless sensor networks (WSNs) form a potential technology for ubiquitous indoor localization due to their autonomous nature, low power consumption [17], and small size factor [18]. The existence of WSNs is enabled by the recent advances in wireless communications and electronics [19]. A WSN may consist of a very large number of small sensor nodes [17], which gather information from their environment by various sensors, process the collected information, control actuators, and communicate wirelessly with each other. Due to the very large number of nodes, frequent battery replacements and manual network configuration are inconvenient or even impossible. Thus, the networks must be self-configuring and self-healing, and the nodes must operate with small batteries for a lifetime of months to years $[17,20]$. This results in very scarce energy budget and constrained data processing, memory, and communication resources.

The usage of the radio transceiver as localization HardWare (HW) is an attractive choice due to its dual-use possibility and inherent existence in WSN nodes. Typically, localization can be performed by measuring signal strengths from the transmissions of neighbors. However, the most lowcost and low-power radio transceivers do not include such a possibility.

Low-power ad hoc medium access control (MAC) protocols have come into existence upon the emergence of WSNs. A radio transceiver is the most power consuming component 
in a WSN node [21]. WSN MAC protocols achieving the lowest power consumption minimize radio usage by accurately synchronizing transmissions and receptions with their neighbors. Typically, the protocols are designed for relatively static network environment, and the energy-efficiency of mobile nodes is degraded. This is problematic in localization point of view, since many located objects can be mobile.

As nodes are moving, their network neighborhood changes introducing increased amount of neighbor discovery attempts. In current WSN MAC proposals, the neighbor discoveries are typically performed by energyhungry network scans requiring relatively long channel receptions. Energy-efficient neighbor discovery protocol (ENDP) [21] introduces a feasible and low-power solution for neighbor discovery. However, also ENDP necessitates a network scan at a start-up and when all communication links to neighbors are broken. The situation is difficult, when a node moves away from the range of other nodes resulting in frequent network scanning. Moreover, to achieve the best possible localization accuracy, mobile nodes need to update measurements frequently from as many neighbors as possible. In current protocols, this necessitates frequent channel reception at the cost of high energy consumption.

Our design aims to achieve ubiquitous real-time localization with low-cost resource constrained nodes. The location nodes are localized using single-hop ranging measurements. The localization data is forwarded via a multihop anchor node network. The presented design builds on top of following objectives and requirements.

(i) Location node energy-efficiency. To enable ubiquitous localization, the location nodes need to run unattended for years with small batteries. Thus, they should reach high energy-efficiency. Anchor nodes are static and considered to be energy unconstrained. Such an assumption is valid, for example, in the field of infrastructure WSNs [22]. Practically, this means that the anchor nodes are mains-powered or equipped with large enough batteries. Location nodes can be highly mobile or relatively static. In either case, the one-hop anchor node neighbors should be reached without considerable increase in power consumption. In addition, a location node can be removed from the anchor network coverage area for undetermined time periods. This should not result in actions reducing energy-efficiency. Addressing the above concerns necessitates the minimization of location node radio usage and MCU active time. Also, energy-inefficient neighbor discoveries should be mitigated.

(ii) Scalability. High densities of location nodes can coexist in the same physical area. Thus, recognizing congestion and adapting to it is an essential demand for the utilized MAC. The spatial scalability of a localization network is highly dependent on the capabilities of the used protocols. In addition, practical issues such as ease of deployment and device costs are in central position. (iii) Bidirectional communication between location nodes and anchor nodes. The ability to communicate with location nodes enables protocol cooperation and expands application-level design space significantly. At communication protocol level, distributed algorithms such as data aggregation, fusion, and cooperative localization are made possible. Applicationlevel issues include, for example, monitoring and user interaction. A location node may integrate sensors with which it can monitor its environment and/or the object or person it is attached to. Furthermore, a person with a location node may send predefined messages and read status information by using a simple user interface (UI) provided by the location node or a device connected to the location node. Applications requiring reliable communication should be taken into account when designing the data transfer support.

(iv) Hardware constraints. For feasible implementation on low-cost, and thus, resource constrained HW platforms, the used protocols should strive for low complexity. Low-cost radio transceivers may not include received signal strength indicator (RSSI), but usually the selection of different transmission power levels is possible. Limited HW capabilities lead to limited ranging information, which the location estimation algorithm has to tolerate.

(v) Accuracy. Our goal is not to improve upon the accuracy of the related RF-based localization approaches, but rather to achieve similar results with very low energy consumption and complexity of nodes. Due to the inaccuracy of the used ranging method we have targeted to the scale of few meters and to good precision in room-level accuracy.

(vi) Real-time operation. In order for location data to be useful, it usually needs to be obtained in real-time. Real-time operation requires low data forwarding latencies from the localized objects to the place of exploitation.

To address the aforementioned objectives and requirements, we present

(i) a novel MAC protocol, called localization MAC (LocMAC), which enables highly energy-efficient location nodes and low-latency multihop data relay, and

(ii) a novel lightweight location resolver algorithm capable of estimating locations using coarse grained and unreliable RF transmission power measurements.

For achieving high energy-efficiency and minimizing resource usage, LocMAC is tightly cross-layer designed with the location resolver algorithm. It includes a built-in support for energy-efficient RSSI-free ranging. The location nodes are relieved from doing neighbor discoveries by an asymmetric protocol approach. The location resolver algorithm employs a novel learning-based transmission power to 


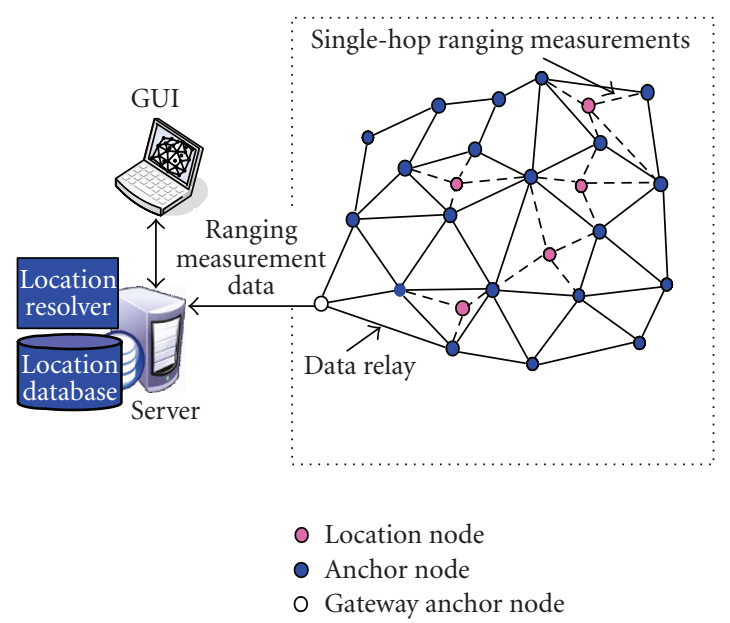

FIgURE 1: Centralized localization network architecture.

distance mapping technique. The scheme is fully calibration free making ad hoc deployment feasible.

The operation of LocMAC is verified by analytical performance analysis, comparison with low-power WSN MAC proposals, and simulations. The feasibility of the proposed localization scheme is validated by experimental measurements using real WSN prototypes. A centralized approach depicted in Figure 1 is utilized. However, centralized operation is not a fundamental constraint to our scheme. Thus, we will also briefly discuss the minor changes needed to make the proposed solution distributed and cooperative.

The key contributions of this paper are

(i) the design of LocMAC and the location resolver algorithm,

(ii) comparison against current state-of-the-art lowpower MAC protocols and RF-based indoor localization proposals, and

(iii) experiments with real resource constrained WSN prototypes.

The rest of the paper is organized as follows. In Section 2, we survey related indoor localization approaches, lowpower WSN MAC protocols, and WSN MAC mobility support. Section 3 describes the LocMAC design. The location resolver algorithm is presented in Section 4. In Section 5, LocMAC is analyzed mathematically and with simulations. Furthermore, LocMAC is compared against state-of-the-art WSN MAC proposals. In Section 6, prototype implementation, experiments, and results are presented. A comparison against related localization proposals is presented in Section 7. Section 8 discusses localization decentralization and optimization issues, and outlines our future work. Finally, Section 9 concludes the paper.

\section{RELATED RESEARCH}

Ubiquitous localization has been widely studied during the recent years. In general, the solutions focus on finding effective location estimation algorithms and measurements that correlate with location. In these designs, medium access has either low priority or it is not considered at all. A detailed survey on ubiquitous localization approaches and taxonomy is given by Hightower and Borriello in [23].

The emergence of WSNs has generated a large amount of MAC protocols aiming for energy- and resource-efficient operation. Their design principles incorporated with localization data acquisition can enable a whole new set of applications.

\subsection{Ubiquitous indoor localization}

Localization approaches can be categorized to range-based, proximity-based, and scene analysis. The underlying technologies vary from pure RF-based, to UltraSound (US), InfraRed (IR), and multimodal solutions.

Range-based approaches rely on estimating distances between location nodes and anchor nodes. This process is called ranging. Received signal strength (RSS) is a common RF-based ranging technique. Distances estimated using RSS can have large errors due to multipath signals and shadowing caused by obstructions $[24,25]$. The inherent unreliability has to be addressed in the used localization algorithms. In $[26,27]$ RSS is replaced with multiple varying power level beacon transmissions. To reduce quantization error, the amount of used transmission power levels is relatively large.

Several location estimation techniques can be used in range-based localization. Utilized methods include trilateration [27, 28], weighted center of gravity calculation [29], and Kalman filtering [9]. Many mathematical optimization methods, such as the steepest descent method [8], sum of errors minimization [26], and minimum mean square error (MMSE) method [30], have been used to solve range-based location estimation problems.

Proximity-based approaches exploiting RF signals $[1,10$, 11, 31] estimate locations from connectivity information. Such solutions are also commonly referred to as range-free in the literature. In WLANs, mobile devices are typically connected to the access point (AP) they are closest to (in signal-space). In the strongest base station method $[10,11]$, the location of the node is estimated to be the same as the location of the AP to which it is connected. In [1, 31 ], the unknown location is estimated using connectivity information to several anchor nodes. Only a very coarsegrained location can be estimated using the strongest base station method. The solutions presented in $[1,31]$ better the granularity to some degree. Nevertheless, in order to reach small granularities the connectivity-based schemes require a very dense grid of anchor nodes. Their strength is fairly simple implementation and modest HW requirements.

Scene analysis consists of an offline learning phase and an online localization phase. The offline phase includes recording RSS values corresponding to different anchor nodes as a function of the users location. The recorded RSS values and the known locations of the anchor nodes are used either to construct an RF-fingerprint database [11, $12,32]$, or a probabilistic radio map [13-16, 33]. In the online phase, the location node measures RSS values to 
different anchor nodes. With RF-fingerprinting, the location of the user is determined by finding the recorded reference fingerprint values that are closest to the measured one (in signal space). The unknown location is then estimated to be the one paired with the closest reference fingerprint or in the (weighted) centroid of $k$-nearest reference fingerprints. Location estimation using a probabilistic radio map includes finding the point(s) in the map that maximize the location probability.

The applicability and scalability of scene analysis approaches are greatly reduced by the time-consuming collection and maintenance of the RF sample database. Searching trough the sample database or radio map is computationally intensive. The joint clustering (JC) technique [15] uses location clustering to reduce the computational cost of searching the radio map. It betters the scalability of the searching algorithm to some extent. MoteTrack [32] achieves similar effect by disseminating the RF-fingerprint database to an WSN and decentralizing the localization procedure.

The described RF-based approaches [1, 26-33] can be considered to utilize networks with WSN characteristics. Due to the autonomous operation of WSNs, the anchor network installation is easy. Used nodes are small and of low-cost thus enabling a cost-effective solution for localization. Since WSNs aim to maximize energy-efficiency, battery-operated nodes hold the potential to achieve long lifetimes.

WLANs are utilized in [8-16]. They are becoming more and more popular offering increased availability. Accordingly, the strength of WLAN-based localization schemes lies in their ability to leverage existing network infrastructure. The costs are increased since every located object must be equipped with a relatively expensive WLAN adapter. WLANs are designed primarily to optimize throughput. Energyefficiency is a secondary objective leading to shortened node lifetime.

In general, RF signal strength-based localization possesses fundamental limits due to the unreliability of the measurements [14]. There is strong evidence that, at best, accuracy in the scale of meters can be achieved regardless of the used algorithm or approach [14].

US-based approaches, namely Active Bat [34] and Cricket [35-37], use time-of-flight ranging and can achieve high accuracies. However, anchor nodes need to be positioned and orientated carefully due to the directionality of US and the requirement for Line-of-Sight (LoS) exposure. A dense network of anchor nodes is needed due to the LoS requirement, short range of US, and the fact that typically ranging measurements to at least four anchor nodes are needed. The addition of US transmitters and receivers increases HW costs and reduces energy-efficiency compared to purely RF-based solutions. Some schemes $[34,36]$ require multiple US transmitters/receivers per one HW platform further increasing the HW costs.

IR-based solutions, such as Active Badge [38, 39], are based on inferring proximity. They can localize location nodes inside the range of LoS IR transmissions. IR-based schemes suffer errors in the presence of obstructions. Also, differing light and ambient IR levels, caused by for example fluorescent lighting or direct sunlight, produce difficulties $[23,35]$. The anchor network costs are high because a dense matrix of IR sensors is needed in order to avoid dead spots.

In the presence of a myriad of location sensing techniques, data fusion has become an attractive location estimation method. It can combine measurements from multiple sensors while managing measurement uncertainty. In [40], Fox et al. survey Bayesian filtering techniques capable of multisensor fusion. Probabilistic fusion methods require relative large amounts of computation. Thus, in the presence of resource constrained nodes, a centralized implementation running in a more powerful base station is often the only feasible choice. For example, in the localization stack [41], the fusion layer is implemented in Java.

Our previous research [42] presents a transmission power -based path loss metering method, that does not require RSSI functionality. The work presented in this paper extends the described method to node localization. Our work differs from RSSI-free approaches $[26,27]$ in three ways. First, our location estimation algorithm is much less computationally intensive than the ones used in $[26,27]$. Secondly, the coarse-grained nature of RSSI-free ranging is accounted for instead of using the signal measurements as traditional ranging measurements with possibly larger quantization error. Third, our approach can cope with any amount of transmission power levels. More importantly, the transmission power level amount can be much lower than with $[26,27]$. In general, the current localization approaches rely on existing communication protocols. This leads to inefficient performance especially in mobile scenarios.

\subsection{Low-power medium access}

Next, we introduce the operation principles of low-power WSN MAC protocols and survey key proposals in the area. Since these protocols are usually designed for relatively static networks, dynamics, especially mobility, can introduce significant additional energy consumption to their operation. The mobility support for WSN MAC protocols is covered in the latter part of the section.

\subsubsection{MAC protocols}

WSN MAC energy-efficiency is achieved by duty-cycling, which includes active periods for data exchanges and sleep periods for energy conservation. WSN MAC protocols can be divided into three categories: random-access, scheduled contention-access, and Time Division Multiple Access (TDMA). The low duty-cycle random-access MAC protocols, such as WiseMAC [22, 43], B-MAC [44], SpeckMAC [45], X-MAC [46], and SCP-MAC [47], are based on a technique called low-power listening (LPL) (name preamble sampling [48] is also used for a method identical to LPL). It includes the procedure of periodically polling the wireless channel to test for traffic. Typically, frames are transmitted with a preceding preamble that is longer than the channel poll interval. This ensures that the destination node is awake during the actual data transmission. Low duty-cycle random-access MAC protocols are relatively simple and 
require less memory than scheduled contention-access and TDMA-based low-duty cycle MACs [21]. Their energyefficiency is reduced due to high idle listening times (caused by frequent channel sampling), and high overhearing. The long preamble presents significant energy costs to the transmission and reception of frames if not mitigated.

Scheduled contention-access low duty-cycle MAC protocols, namely S-MAC [49], T-MAC [50], and IEEE 802.15.4 low-rate wireless personal area network (LR-WPAN) standard [51], utilize periodic active and sleep periods to achieve duty-cycling. The start of the active period includes the transmission of synchronization (SYNC) frames to communicate own schedule information to neighboring nodes. The rest of the active period is reserved for data exchanges, which typically use contention-access for medium arbitration.

TDMA-based low duty-cycle MAC protocols, including SMACS [52], LEACH [53], PACT [54], TRAMA [55], SRSA [56], and TUTWSN MAC [57], exchange data only in predetermined synchronized time slots. Rest of the time is spent in sleep mode. This makes the protocols virtually collision-free and removes overhearing. The only sources of idle listening are reception margins, which are usually relatively small. In static networks, TDMA-based MAC protocols can achieve even an order of a magnitude lower energy consumption than low duty-cycle random-access and scheduled contention-access MACs $[45,57]$.

\subsubsection{Mobility support}

As network dynamics increase, neighbor discovery starts to produce significant energy overhead with synchronized low duty-cycle MAC protocols, which include all scheduled contention-access and TDMA-based protocols and SCPMAC from low duty-cycle random-access protocol family. The rest of the low duty-cycle random-access protocols are unsynchronized and do not require explicit neighbor discovery.

Network scanning is the typical mechanism for neighbor discovery in current low-power MAC proposals [21]. It may consume energy equal to the transmission of thousands of data packets [58].

The term network scanning refers to the generic procedure of continuous listening for neighbors' control data until sufficient knowledge of the neighborhood is obtained. This requires the listening to go on for the duration of a synchronization period. Thus, the term network scanning is applicable to the following procedures:

(i) listening for in-channel signaling messages possibly on one or many RF frequency channels, as in SCPMAC, S-MAC, T-MAC, and IEEE 802.15.4,

(ii) listening for out-of-channel signaling messages on a network-wide fixed signaling channel, as in SMACS and TUTWSN MAC, and

(iii) listening for signaling messages during a periodical signaling period, as in LEACH, PACT, TRAMA, and SRSA.
Several studies, such as $[59,60]$, address mobility and the energy constraint at the network layer. However, work aiming to improve energy-efficiency under mobility at the MAC layer is more rare. Since majority of energy overhead is caused by idle listening at the data link layer, significant energy saving can be achieved by addressing mobility in the MAC protocol [61].

Mobility-aware Sensor MAC (MS-MAC) [61] is based on S-MAC. It adjusts network scan interval according to the mobility of nodes. Mobility-adaptive MAC (MMAC) [62] works similarly as MS-MAC, but it uses TRAMA as the basic MAC protocol and adjusts the occurrence frequency of the signaling period according to mobility. These approaches allow mobile nodes to acquire new connections more efficiently and reduce packet losses. However, the energy consumption is high due to frequent networks scans.

Raviraj et al. [63] propose an adaptive frame size predictor to overcome the energy-inefficiency caused by frame losses in mobile scenarios. The approach can improve energy-efficiency by using smaller frame sizes when the wireless channel characteristics are poor. However, the method does not consider neighbor discovery, and thus, mitigates only the energy-inefficiency caused by larger bit error rate due to mobility and Doppler shifts.

From the covered low duty-cycle MAC protocols, only SMACS consider mobility explicitly. For mobile nodes, it proposes an Eavesdrop-And-Register (EAR) algorithm. The EAR algorithm is designed to save energy for stationary nodes in the presence of mobile nodes. Mobile nodes must listen almost constantly resulting in high energy consumption. Thus, the algorithm is not applicable with energy-constrained mobile nodes.

Our concurrent work, ENDP [21], reduces the need for costly network scans by proactively distributing node schedule information. Two-hop neighborhood synchronization information is piggybacked in beacon transmissions. ENDP can achieve low energy consumption when continuously having at least one working link. At a start-up and when all communication links to neighbors are broken, also ENDP has to fall back on network scanning. To the best of our knowledge, ENDP is currently the most energy-efficient neighbor discovery protocol for dynamic WSNs using low duty-cycle MAC protocols.

The work presented in this paper achieves energyefficient mobile location nodes by relieving them from doing neighbor discoveries. For this, an asymmetric architecture, where energy unconstrained anchor nodes listen almost continuously, is used. The location node energy-efficiency is independent of the scenario and environment.

\section{LOCMAC DESIGN}

LocMAC is comprised of two subprotocols; LocMAC base and LocMAC relay. LocMAC base enables energy-efficient single-hop localization data acquisition. It arbitrates the wireless medium between location nodes. It can be integrated to any data gathering network, whose operation includes idle times. By giving the data gathering network protocols higher priority and executing LocMAC base in 
a separate channel, their operation is not affected. Thus, LocMAC base does not dictate the usage of LocMAC relay.

LocMAC relay provides localization data aggregation and low-latency data relay. It is primarily designed for multihop data gathering using LocMAC base as the data source. Thus, some parts of its functionality require the existence of LocMAC base.

\subsection{Energy-efficient localization data acquisition}

LocMAC base enables energy-efficient location node operation. In the rest of this section, we will focus on its operation principles.

\subsubsection{Location node channel access and collision avoidance}

LocMAC base uses duty-cycling to achieve energy-efficiency. Location node time is divided into active periods and idle periods. A location node starts its active period by transmitting $N_{\mathrm{lb}}$ location beacons (LB), which constitute a beacon set. Energy unconstrained anchor nodes use their idle time listening for LBs in the location channel. They acknowledge received LBs in a downlink slot following the beacon set using a location beacon acknowledgement (LBA) frame. Consecutive active and idle period constitutes a beacon cycle, which is repeated at interval $T_{\mathrm{bc}}$. By making anchor nodes scan for LBs, the location nodes are relieved from performing active neighbor discovery.

The LBs are dual-purpose. First, they form the basis of LocMAC base collision-avoidance (CA) mechanism with the LBAs. Concurrently, LBs enable coarse-grained RSSI-free RF-based ranging.

Only one downlink slot is used so that the energy consumption and the time one location node occupies the location channel would be minimal. The usage of one downlink slot requires an arbitration mechanism in order to avoid multiple anchor nodes transmitting in the same slot. A reservation-based slot allocation mechanism is infeasible because of high expected network dynamics and short-lived links. Thus, simple randomization is used to control who is allowed to transmit in the downlink slot.

LocMAC base divides time into discrete time slots referred to as active period slots. Ideally, each active period slot should contain the active period of only one location node. Since location nodes access the channel asynchronously, their active period slot boundaries do not occur at same time instants. Thus, the active period slot length is set to be two times as long as the active period duration and the active period occurs at the middle of the slot. This ensures that an active period occurring in a randomly selected slot in the schedule of location node $x$ can collide with only one active period in the schedule of location node $y$.

Figures 2 and 3 illustrate LocMAC base operation principle. They present two location nodes, 11 and 12, and two anchor nodes, 1 and $2 . N_{\mathrm{lb}}$ is set to four. Transmission powers are enumerated with integers from 1 to 4,1 indicating the lowest and 4 the highest transmission power. $\mathrm{LB}_{n}$ denotes

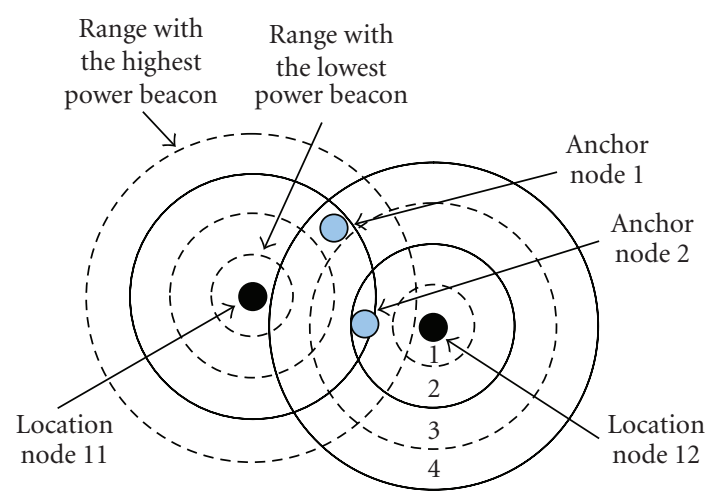

FIGURE 2: LocMAC base operation principle: relative locations of anchor and location nodes and radio ranges with different transmission powers.

a location beacon transmitted with power level $n$. The node locations relative to each other and relative to radio ranges are illustrated in Figure 2. In the depicted scenario, location nodes infer overlapping active periods from two or more missed LBAs. Due to space limitations in Figure 3, the active period slot length is equal to the active period duration.

At epoch $\mathbf{A}$ in Figure 3, location node 12 is able to successfully transmit $\mathrm{LB}_{2}$ to anchor node 2 (no overlap in time) and $\mathrm{LB}_{4}$ to anchor node 1 (no overlap in radio coverage). $\mathrm{LB}_{3}$ of location node 11 collides with the downlink slot of location node 12 . Location node 11 still succeeds to transmit $\mathrm{LB}_{4}$ to both anchor nodes. However, the anchor nodes omit acknowledging it, since they infer possible active period overlap. Epoch B presents similar events as epoch A. After epoch B, both location nodes have missed two LBAs. Thus, the location nodes determine that they have conflicting schedules. After inferring a conflicting schedule, a location node chooses a new one by randomizing a new active period slot.

The new active period slot is randomized between interval $\left[t_{\text {aps_end }(k)}, t_{\text {aps_start }(k+2)}\right]$, where $t_{\text {aps_end }(k)}$ is the end time of current active period slot $(k)$ and $t_{\text {aps_start }(k+2)}$ is the start time of active period slot $k+2$. The randomization interval end is given by

$$
t_{\text {aps_start }(k+2)}=t_{\text {aps_end }(k)}+2 T_{\mathrm{bc}}-t_{\mathrm{aps}} \text {, }
$$

where $T_{\mathrm{bc}}$ is the beacon cycle length and $t_{\mathrm{aps}}$ is the active period slot length. After a location node has randomized a new slot, it adjusts its beacon cycle length to $T_{\text {bc_adj }}$ for one beacon cycle in order to adapt to the new schedule. After the adaptation, the location node starts using the normal beacon cycle length $\left(T_{\mathrm{bc}}\right)$ again.

The randomized slot index can be between $[-\operatorname{sid} x$, $\operatorname{sid} x]\left(\operatorname{sid} x \in \mathbb{N}_{+}\right)$, where $\operatorname{sid} x=N_{\text {rnd_slots }} / 2-1$ and index 0 denotes a slot that would result in no adjustment (i.e., $\left.T_{\text {bc_adj }}=T_{b c}\right)$. $N_{\text {rnd_slots }}$ denotes the amount of randomization slots. Equal probability to temporarily adjust beacon cycle shorter or longer results in

$$
T_{\mathrm{bc}}=\lim _{n \rightarrow \infty} \frac{\sum_{k=0}^{n}\left(t_{\mathrm{bc}(k+1)}-t_{\mathrm{bc}(k)}\right)}{n},
$$




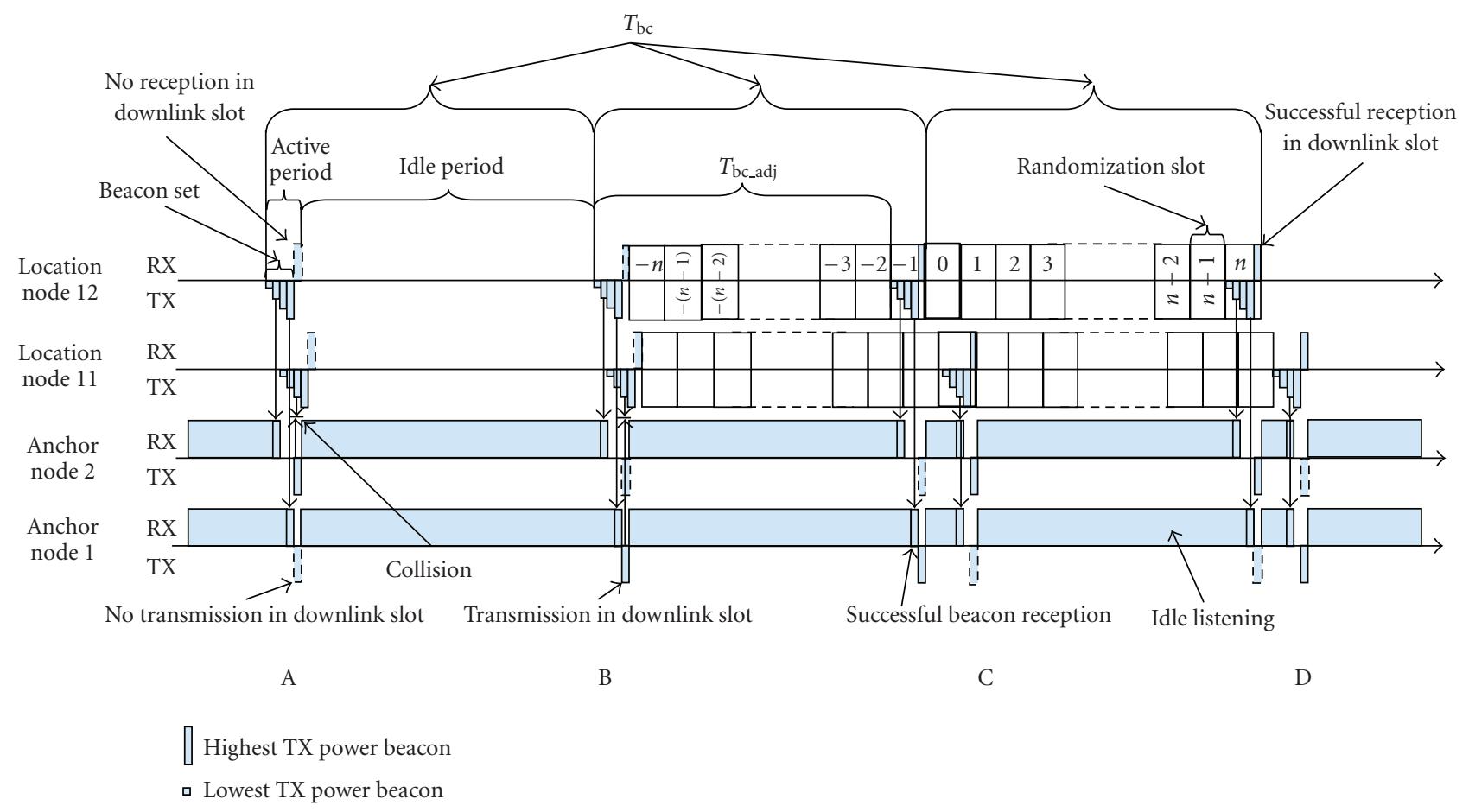

FIGURE 3: LocMAC base operation principle: LocMAC beacon cycle components, collision avoidance, and timing.

where $t_{\mathrm{bc}(k)}$ is the start time of beacon cycle $k$. Thus, the mean beacon cycle length is always $T_{\mathrm{bc}}$, which results in fair channel access and predictable energy consumption.

In Figure 3 at the end of epoch B, location node 12 randomizes its active period to slot -1 and location node 11 to slot $0(\operatorname{sid} x$ being $n)$. At epoch $\mathbf{C}$, the new schedules are adopted, resulting in a collision-free situation. Now both location nodes can successfully transmit their LBs and receive corresponding LBAs. Location nodes continue with same schedules at epoch $\mathbf{D}$ since they infer nonconflicting situation from successful LBA receptions.

\subsubsection{Detecting and handling false location beacons}

A false LB reception occurs when an anchor node is situated in the range of an LB transmitted with power $P$, but it can only hear $\mathrm{LB}(\mathrm{s})$ transmitted with power that is larger than $P$. This situation can occur if

(i) an anchor starts listening the location channel in the middle of an active period and/or

(ii) active periods of two or more location nodes overlap partially.

Detecting falsely observed LBs has two benefits. First, it serves as an indicator for partly overlapping, and thus, conflicting active periods, as happens in Figure 3 at epochs A and B. Secondly, the location estimation algorithm can be informed of invalid input data.

When an anchor node starts listening the location channel in the middle of an active period, a possibly false $\mathrm{LB}$ can be detected by counting the time passed between the listening start time $\left(t_{\text {st_start }}\right)$ and the reception time instant of the $\mathrm{LB}\left(t_{b}\right)$. The LB can be false if

$$
t_{b}-t_{\mathrm{lst} \_s t a r t}<t_{\mathrm{bs}}
$$

where $t_{\mathrm{bs}}$ is the beacon set length.

When active periods of two or more location nodes overlap partially, a possibly false LB can be detected by observing the time passed between the end of the last detected active period $\left(t_{\text {end_ap }}\right)$ and the reception time instant of the current LB $t_{b}$. Thus, an LB can be false if

$$
t_{b}-t_{\text {end_ap }}<t_{\mathrm{bs}} .
$$

\subsubsection{Data transfer}

LocMAC base enables low-rate data exchanges between location nodes and anchor nodes. Both LBs and LBAs include payload parts. Uplink data can be piggybagged in LB frames, while downlink data can utilize LBA frames.

Two quality-of-service (QoS) classes, datagram and reliable, are supported. Datagram protocol data units (PDU) are transmitted once after which they are removed from the PDU queue. Reliable PDUs remain in the queue until they are acknowledged or an application-specific timeout occurs.

\subsubsection{Scalability}

The LB/LBA CA mechanism enables spatial scalability by handling the hidden node problem [64] similarly to CTS/RTS mechanism in IEEE 802.11 CSMA/CA [65]. Collisions at the receiver (anchor node) are inferred from the absence of acknowledgments (LBAs). 
The active period slot randomization (APSR) mechanism handles scalability in location node amount. The absolute theoretical maximum amount $\left(N_{\text {ln_max }}\right)$ of coexisting location nodes in the same radio coverage area is given by

$$
N_{\text {ln_max }}=\left\lfloor\frac{T_{\mathrm{bc}}}{t_{\mathrm{aps}}}\right\rfloor .
$$

When the location node amount in the same radio coverage area starts to approach $N_{\text {ln_max }}$, the LocMAC base CA performance will hinder due to congestion. Location nodes adapt to congestion by increasing their beacon cycle lengths $\left(T_{\mathrm{bc}}\right)$, which in turn leads to increased $N_{\text {ln_max }}$.

In order to reduce interference range, an LBA frame is always transmitted with the same transmission power as the minimum received $\mathrm{LB}$. By monitoring the received LBA transmission powers, location nodes can gain information about the amount of anchor nodes they can reach using a certain transmission power. In the presence of excess anchor nodes, the maximum LB transmission power can be scaled down reducing the beacon set interference range. The downscaling of the transmission power results also in energy savings, and shorter active period.

\subsection{Low-latency data relay}

LocMAC relay enables the anchor nodes to establish a network for multihop data forwarding. It exploits both TDMA and Frequency Division Multiple Access (FDMA) to share the medium. The medium has to be arbitrated among the relay nodes and between relay network and the location nodes.

LocMAC relay specifies only the medium access. For routing, for example a spanning tree algorithm resolving minimum-hop routes to the sink can be used.

\subsubsection{Network topology}

The LocMAC relay protocol exploits flat relay topology superpositioned with a clustered data aggregation topology. An example is illustrated in Figure 4. The relay network consists of relay nodes and single or multiple sinks. The relay nodes form a flat topology to enable data forwarding to the $\operatorname{sink}(\mathrm{s})$. For data aggregation, clusters are established. Each aggregation cluster consists of an aggregation cluster head $(\mathrm{ACH})$ and $n$ subnodes acting as cluster members. At data relay level relay nodes are homogenous.

The ACHs act as data aggregation points. Data aggregation is performed on measurements belonging to the same beacon set. Location node ID and beacon set transmission timestamp (either local or global time can be used for the unique beacon set identifier formation) can be used to form a unique identifier for measurements belonging to the same beacon set. After the aggregation point, a flat topology is used and subsequent data relaying and routing can be done by any node in the relay network.

In Figure 4, the LBs transmitted by location node 1 are heard by relay nodes 2, 3, and 4 . Nodes 2 and 3 act as subnodes. They forward LB information to their ACH, node

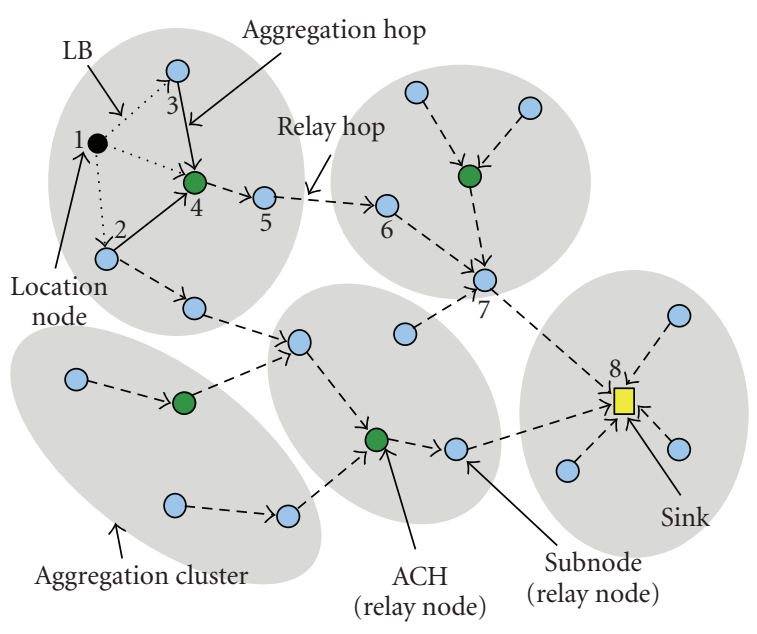

FIgURE 4: Relay network topology.

4. Node 4 aggregates the data it has received directly from the location node and the data relayed by its members. Then it forwards the aggregated data towards the sink (node 8 ). The data is relayed via nodes 5, 6, and 7 .

\subsubsection{Autonomous network build-up and maintenance}

Neighbor discovery is enabled by network beacons, which all relay nodes transmit in a common network-wide channel. A separate channel is used to reduce interference with the location channel. The network beacon transmissions are scheduled by randomizing the transmission interval $\left(T_{\mathrm{nb}}\right)$ between $T_{\text {nb_min }}$ and $T_{\text {nb_max }}$. The randomization prevents sequential beacon collisions. $T_{\text {nb_min }}$ can be used to reduce congestion in the network channel. $T_{\text {nb_max }}$ gives a theoretical upper bound for discovering all neighbors in the same radio coverage area, and limits the maximum network scan length. The network beacons are transmitted with varying transmission powers to enable link quality monitoring without RSSI. To enable clustering, node role $(\mathrm{ACH}$ or subnode) is indicated in the network beacons.

A neighbor discovery is performed with a network scan. After the scan, a node will have a list of neighbors represented by tuples in the form of $\left\langle\mathrm{ID}_{i}, Q_{i}, L_{i}\right\rangle$, where $\mathrm{ID}_{i}$ is the ID, $Q_{i}$ is the link quality, and $L_{i}$ is the load of relay node $i$. A subnode chooses an ACH $i$ whose parameters $Q_{i}$ and $L_{i}$ minimize cost function $c(Q, L)$. The cost function is of form

$$
c(Q, L)=a \frac{1}{Q}+b L
$$

where $a$ and $b$ are weighting factors for converting link quality and load to cost values. Better link quality reduces transmission failures, and smaller load lowers latency. Parameters $a$ and $b$ are network-specific constants.

Upon choosing a minimum cost $\mathrm{ACH}$, a subnode backs off for a randomized amount of time. After the backoff time, it transmits an association request to the chosen $\mathrm{ACH}$. The backoff procedure is used to avoid collisions 
among association request packets. The location channel is used for the association data exchange, since relay nodes are already listening on it for LBs. The association data exchanges happen infrequently. Thus, minimal interference is inflicted upon the actual localization data acquisition. Upon receiving an association request, an $\mathrm{ACH}$ responds with an association response. If the association is successful, the response contains the allocated TDMA time-slot index used for communication between an $\mathrm{ACH}$ and associated subnodes. The slots are assigned in the same order as the association requests are received.

Relay nodes may fail or be removed from the network and new ones may be added. To maintain up-to-date neighbor information, nodes do periodical network scans. If an $\mathrm{ACH}$ is lost, its members scan the network and associate to new ACHs. Subnodes do periodical reassociations, which enable ACHs to detect subnode failure and release corresponding allocated slots.

The roles of ACHs and subnodes can be either selected manually or a dynamic cluster-head election algorithm, such as is presented in [53], can be used. This is merely an implementation-specific issue and not dictated by the presented design.

\subsubsection{Channel access}

Relay network data exchanges can be divided into intracluster aggregation data exchanges and to relay data exchanges. The aggregation data exchanges occur between ACHs and their members. The relay data exchanges take place between nodes in the relay path to the sink. For both data exchange types, a different kind of channel access scheme is used.

The aggregation data exchanges occur after nodes in a cluster have received LBs from a location node (Figure 5(a)). For the aggregation data exchanges, reservable time slots are used. The time slots form an aggregation superframe as illustrated in Figure 5(b). Each time slot is further divided into an uplink slot and a downlink slot. Reliable communication is enabled using MAC-level acknowledgments.

A synchronization mechanism is needed to correlate a time slot to a common time reference among the nodes. A receiver-based synchronization scheme, similar to reference broadcast synchronization (RBS) [66], is used. Instead of using explicit synchronization packets, LBs are exploited to achieve synchronization.

Upon receiving an $\mathrm{LB}$, an $\mathrm{ACH}$ or a subnode has knowledge about the exact time the active period of the source location node ends. The location node active period end acts as a synchronization point as depicted in Figure 5(b). The aggregation superframe follows the active period of the location node.

The relay data exchanges after aggregation are illustrated in Figures 5(c), 5(d), 5(e), and 5(f). The protocol exploits the fact that the next hop node can overhear the acknowledgment sent to the current hop source node (similar reasoning is made for CTS packets in MARCH protocol [67]). Thus, to reduce signaling and latency, the acknowledgments are made dual-purpose. First, they are used to acknowledge received packets. Secondly, the current hop acknowledgment is used to signal next hop node of upcoming communication (Figures 5(c) and 5(e)).

The relayed data is communicated on separate RF channels. This reduces the interference that the the relay process causes to the localization data-acquisition process. Furthermore, by randomizing the channel for every relay communication transaction separately, the interference between different data relay flows is minimized. The acknowledgments are sent on location channel, so that the next hop nodes are able to receive them during the normal operation of listening for possible LBs. The current acknowledgment contains the randomized RF channel, the next hop node ID, and the exact time for next data relay transmission. Thus, the next hop node knows when to start listening in a correct channel.

\subsubsection{Problematic situations}

To make the channel access robust, the following problematic situations need to be addressed:

(i) an $\mathrm{ACH}$ does not hear any LB, but its members do,

(ii) subnodes belonging to the same cluster hear LBs from different location nodes, and

(iii) acknowledgment packets collide.

The first situation is resolved by using the location channel for aggregation data exchanges. If an $\mathrm{ACH}$ does not hear an LB, it cannot infer a synchronization point. Nevertheless, since all idle time is spent listening on the location channel, the relayed frames can be received.

The second situation introduces collisions, if two or more location nodes have active periods temporally close to each other. If subnodes fail to successfully transmit data in the reserved time slots, they try to retransmit the frames using slotted ALOHA [68].

If an acknowledgment packet is lost, the next hop node cannot receive the corresponding relay packet. In this situation, the sender backs off for a random amount of time and tries to resend. The retransmission is signaled using an explicit RTS packet, which includes the same next hop information as an acknowledgment would.

\section{LOCATION RESOLVER ALGORITHM}

The presented location resolver algorithm follows the same principal idea as cell identification (CI) in cellular networks. In cellular networks, mobile stations (MSs) try to connect to a base station (BS) nearest to them. Thus, the MSs can infer their location to be somewhere in the coverage area of the BS in question [3].

Our algorithm can tolerate inaccurate measurements by using novel learning-based transmission power to range mapping method. Furthermore, the algorithm is computationally light, enabling its usage in resource-constrained nodes and making decentralization possible. 


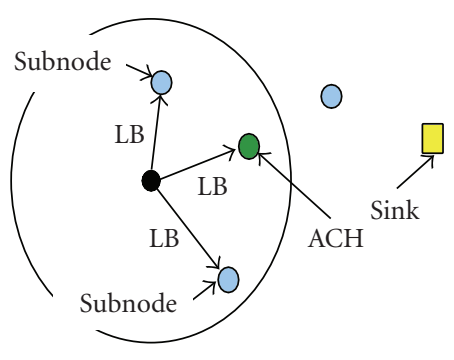

(a) Location node transmits LBs, which are received by an $\mathrm{ACH}$ and two subnodes

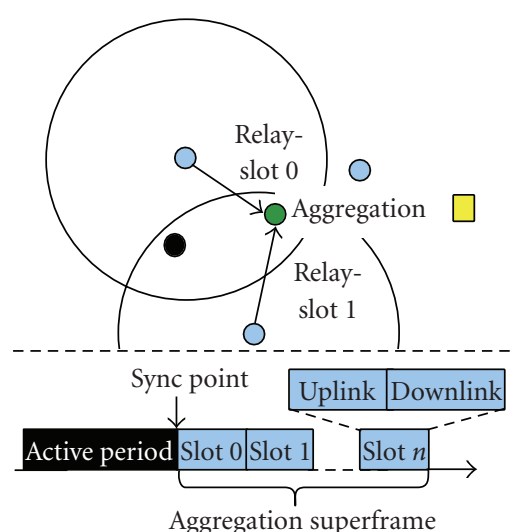

(b) The subnodes relay the measurement data to the ACH they are associated to. After relay data reception, the $\mathrm{ACH}$ performs aggregation

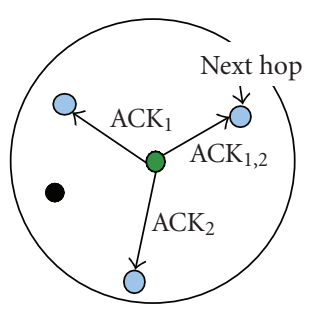

(c) The ACH acknowledges the relayed data packets. The acknowledgements simultaneously signal the next hop node of upcoming data packet

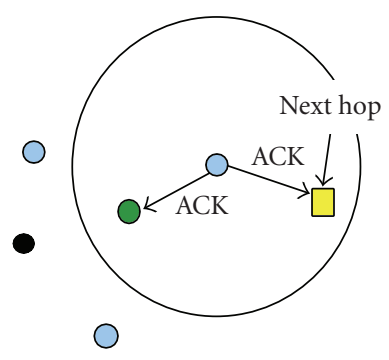

(e) The intermediate node acknowledges the received packet. The acknowledgement simultaneously signals the sink of upcoming data packet

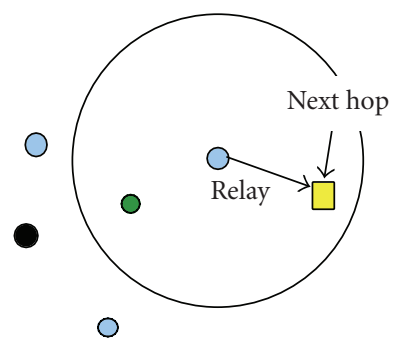

(f) The data packet is relayed to the sink next hop node according to the schedule informed in the last acknowledgement packet

Figure 5: Localization data aggregation and relay via multiple hops to the sink.

\subsection{Location resolution using bounding boxes}

Our scheme uses an approach, where an anchor node radio range with a certain transmission power is considered as a cell. Furthermore, variable transmission powers are used to introduce variable sized cells. The used algorithm tries to find out the minimum area that is bounded by the overlapping minimum-sized cells. To simplify calculations without considerably degrading the accuracy, the cells are modeled to be squares.

Figure 6 depicts three anchor nodes and one location node. The anchor nodes can hear the location node with powers $P_{1}, P_{2}$, and $P_{3}$, which map to radio ranges $r_{1}, r_{2}$, and $r_{3}$, respectively. Furthermore, the radio ranges map to radio coverage circles and square localization cells (LCs). A square can be fully determined by giving the coordinates of its bottom left and top right corners; $\mathrm{LC}_{n}=\left\{P_{\text {bl_LC }(n)}, P_{\text {tr_LC }(n)}\right\}$. The set of LCs used in one location estimation is denoted by LCS. In order to find the final bounding box (FBB) containing the location node, the intersection of LCs in one LCS needs to be determined. FBB is given by

$$
\mathrm{FBB}=\bigcap_{\mathrm{LC}_{n} \in \mathrm{LCS}} \mathrm{LC}_{n}
$$

Equation (7) can be solved by a lightweight algorithm called min-max [69-71]. Min-max relies on the fact that the intersection of all LCs can be acquired by taking the maximum of all coordinate minimums and the minimum of all maximums:

$$
\operatorname{FBB}=\left\{\left(\max \left(X_{\mathrm{bl}}\right), \max \left(Y_{\mathrm{bl}}\right)\right),\left(\min \left(X_{\mathrm{tr}}\right), \min \left(Y_{\mathrm{tr}}\right)\right)\right\},
$$

where $X_{\mathrm{bl}}$ is the set of bottom left $x$-coordinates, $Y_{\mathrm{bl}}$ is the set of bottom left $y$-coordinates, $X_{\mathrm{tr}}$ is the set of top right $\mathrm{X}$ coordinates, and $Y_{\mathrm{tr}}$ is the set of top right $y$-coordinates in LCs contained by LCS.

\subsection{Learning-based transmission power to range mapping}

The raw input data for the localization procedure consists of a set of tuples in the form of $\left\langle p_{i}, P_{i j}\right\rangle$, where $p_{i}$ is the position of an anchor node $i$ and $P_{i j}$ is the transmission power of the minimum power LB received by an anchor node $i$ and sent by a location node $j$. The determination of LCs, which are needed by the bounding box algorithm, requires a procedure for mapping transmission powers $\left(P_{i j}\right)$ to 


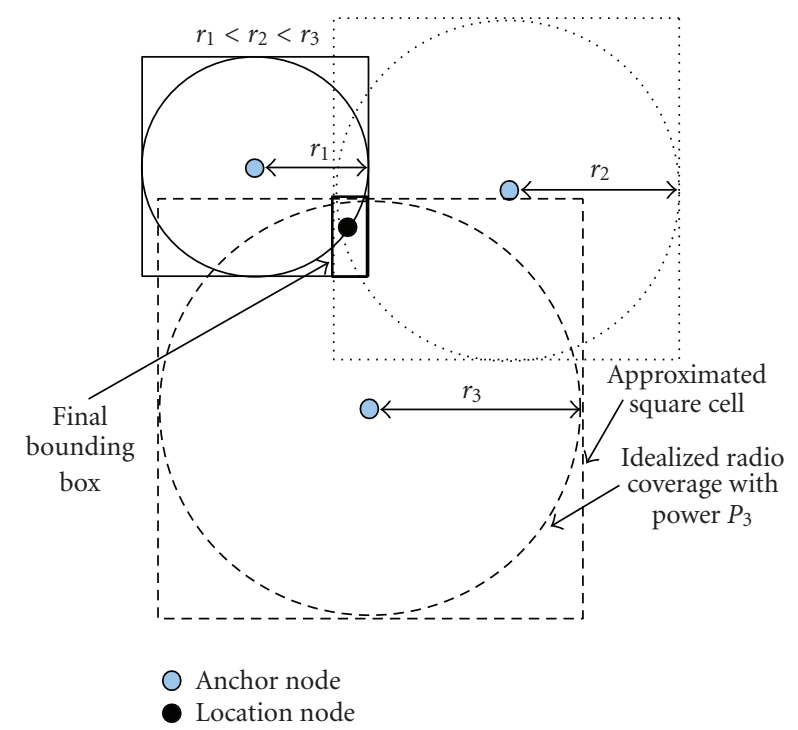

Figure 6: Localization cells and final bounding box.

ranges $\left(r_{i j}\right)$. Usually, an initial calibration and measurements are required in the network setup phase to find out the relationship between RF-power and range. At fixed location, the signal strength received from an anchor node changes with time [15], implying the need for recurrent calibrations $[11,16]$.

We acknowledge that also our location estimation algorithm would benefit from the calibration procedure. However, in order to achieve easy deployment and robust operation in changing conditions, it is not used. Instead, we introduce an algorithm called learning-based transmission power to range mapping. It corrects initial approximated ranges at run time and considers local environmental and RF propagation properties automatically.

Simplified indoor path loss models follow two basic approaches. In the first scheme, an explicit attenuations for every wall is added and the path loss between walls is treated as free-space path loss. Alternatively, the attenuation caused by walls is added implicitly by changing the path loss exponent $(e)$ value. Since our algorithm has no knowledge of the environment and the obstacles contained by it a priori, we have adopted the varying path loss exponent approach.

Learning-based transmission power to range mapping is based on iterative bounding boxes technique. Initially, an approximated path loss exponent value is used. Now, if the real path loss exponent is smaller than the approximated one, the bounding boxes do not necessarily overlap. This situation is depicted in Figure $7(\mathrm{a})$, where $r(P, e)$ gives the range with transmission power $P$ and path loss exponent $e$. The iterative bounding boxes algorithm decrements the path loss exponent (and increments range) until all the bounding boxes in the calculation overlap and a valid result is obtained. This is illustrated in Figure 7(b). The resolved path loss exponent value is saved and can be used in future location resolutions.

If a situation where bounding boxes do not overlap is met again, the iterative bounding boxes algorithm is triggered starting with the current path loss exponent value. This enables the maintenance of the path loss exponent giving the "worst case" LCs, without excessive runs of the iterative algorithm. Figure 8 illustrates the relation of the worst case LC to the ideal and real radio coverage. The use of the worst case LC guarantees that the resolved FBB contains the location node. In order to avoid using the worst case path loss exponent value all the time, it can be reset to its initial value periodically. This way temporal changes in the environment can be taken into account.

Iterative bounding boxes increase the processing overhead compared to the simple bounding boxes algorithm, since $r(P, e)$ needs to be repeatedly calculated. Fortunately, the processing cost is amortized, since after a valid path loss exponent is solved, a pair $\left\langle P_{i}, r_{i}\right\rangle$ has been found. This reduces the rest of the transmission power to distance mapping procedures to simple array indexing operations using $P_{i}$ as the key.

\section{LOCALIZATION DATA ACQUISITION PERFORMANCE ANALYSIS}

In this section, we will first compare the energy-efficiency of LocMAC base against low-power WSN MAC protocols. Then, LocMAC base collision avoidance effectiveness is evaluated using Matlab simulations.

\subsection{Localization data acquisition energy-efficiency comparison}

The related low-power MAC protocols are represented by four ideal protocol models; two contention-based and two schedule-based. First, models applicable for static networks are defined. Then, the models are complemented with neighbor discovery, which is needed when nodes are mobile.

Our scheme is primarily targeted for very simple node HW using a radio transceiver not including RSSI support. This would make the usage of MAC protocols dependant on RSS infeasible. Nevertheless, they are also included to the comparison for thoroughness.

\subsubsection{Derivation of MAC protocol models}

The first contention-based protocol model, referred to as contention-MAC-unsync, represents low duty-cycle random-access MAC protocols. Energy unconstrained anchor nodes can listen continuously to potential uplink traffic (excluding the time they send packets). Thus, a preamble of extended length is redundant when transmitting uplink and nonpersistent CSMA can be used. For energy-efficient downlink communication, contentionMAC-unsync uses LPL. A location node beacon cycle using contention-MAC-unsync is illustrated in Figure 9.

The second contention-based protocol model, referred to as contention-MAC-sync, represents scheduled contentionaccess low duty-cycle MAC protocols. Nonpersistent CSMA is used both to the uplink and the downlink direction. For downlink data, the nodes need to listen the channel continuously for the time of the listen period. A location 


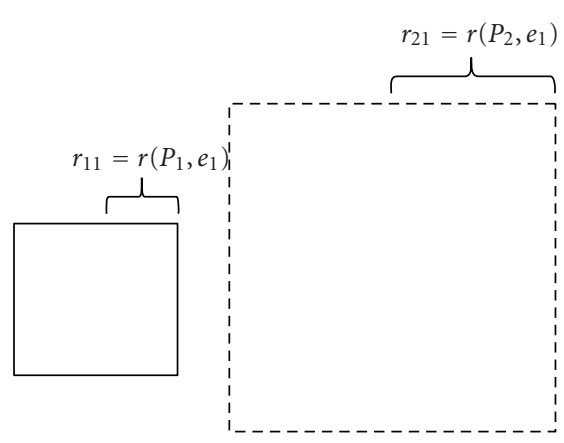

(a)Bounding boxes with initial path loss exponent

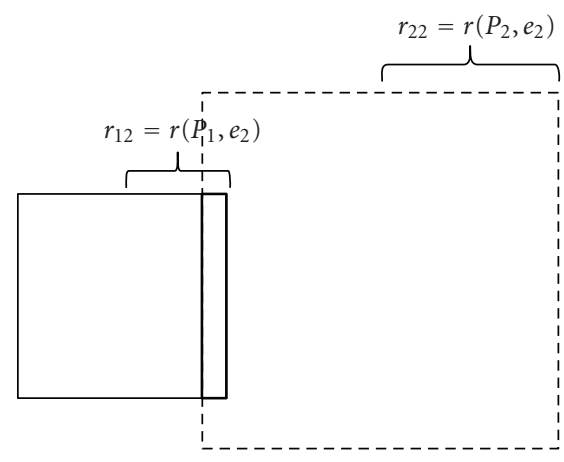

(b) Bounding boxes with final path loss exponent

FIgURE 7: Iterative bounding boxes.

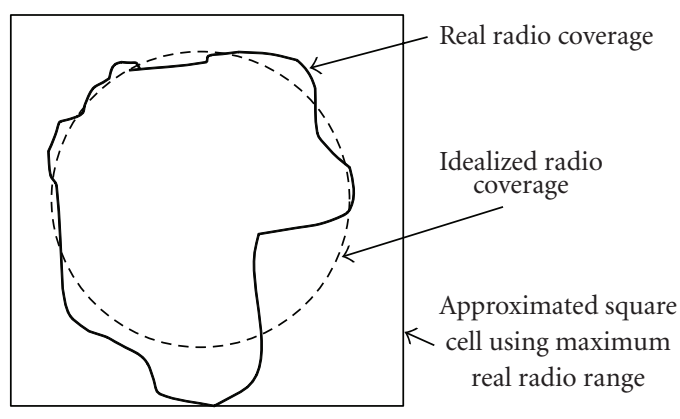

FIGURE 8: Worst-case localization cell.

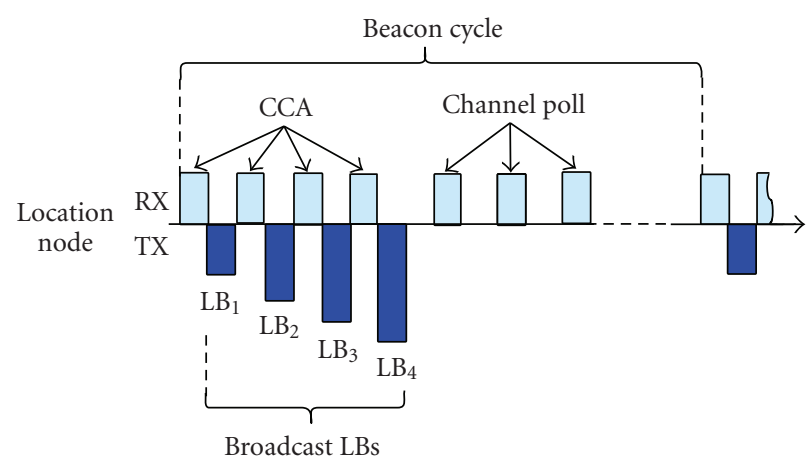

FIgURE 9: Location node beacon cycle using contention-MACunsync.

node beacon cycle using contention-MAC-sync is illustrated in Figure 10.

The first schedule-based protocol, referred to as scheduled-MAC-link, uses TDMA with link activation. slot assignment scheme. Link activation does not enable the usage any single time slot for broadcast purposes. Broadcast has to be established as a series of unicasts as depicted in Figure 11. Thus, the cost of transmitting LBs and listening for downlink data is multiplied by the amount of synchronized neighbors. (There are two commonly used TDMA slot

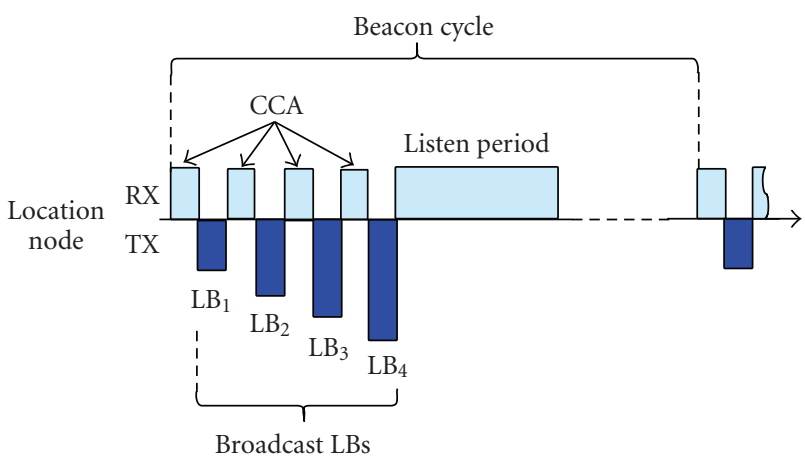

Figure 10: Location node beacon cycle using contention-MACsync.

allocation schemes referred to as node activation and link activation [54]. In node activation slots are assigned to individual nodes. Link activation assigns slots to links. Node activation allows efficient broadcast, since a node is able to transmit to any of its neighbors in its allocated slot. Nodes are not allowed to transmit simultaneously to neighbors that are not common to them even if this would not result in a collision. Link activation presents same properties in reversed order.) The second schedule-based protocol, referred to as scheduled-MAC-node, utilizes TDMA with node activation slot assignment scheme. As can be seen from Figure 12, an LB broadcast reserves only one time slot. The downlink slot amount is still proportional to the amount of synchronized neighbors.

\subsection{2. power consumption models}

Next, power consumption expressions for a location node using contention-MAC-unsync, contention-MACsync, scheduled-MAC-link, scheduled-MAC-node, and LocMAC base are derived. The used symbols, their descriptions, and defined values are summarized in Table 1. Typical values for IEEE.802.15.4 compliant radio (Chipcon CC2420 [72]) and the radio used in our prototype platforms (Nordic Semiconductor nRF24L01 [73]) are given. 
TABLE 1: Symbols, descriptions, and defined values for MAC power consumption analysis.

\begin{tabular}{|c|c|c|c|}
\hline Symbol & Description & CC2420 & nRF24L01 \\
\hline$P_{\mathrm{tx}(n)} n=4$ & Power in transmission mode at $0 \mathrm{dBm}$ & $52.2 \mathrm{~mW}$ & $33.9 \mathrm{~mW}$ \\
\hline$P_{\mathrm{tx}(n)} n=3$ & Power in transmission mode at $-7 /-6 \mathrm{dBm}$ & $37.5 \mathrm{~mW}$ & $27 \mathrm{~mW}$ \\
\hline$P_{\mathrm{tx}(n)} n=2$ & Power in transmission mode at $-15 /-12 \mathrm{dBm}$ & $29.7 \mathrm{~mW}$ & $22.5 \mathrm{~mW}$ \\
\hline$P_{\mathrm{tx}(n)} n=1$ & Power in transmission mode at $-25 /-18 \mathrm{dBm}$ & $25.5 \mathrm{~mW}$ & $21 \mathrm{~mW}$ \\
\hline$P_{\mathrm{rx}}$ & Power in reception & $56.4 \mathrm{~mW}$ & $35.4 \mathrm{~mW}$ \\
\hline$P_{\text {sleep }}$ & Power in sleep mode & $60 \mu \mathrm{W}$ & $2.7 \mu \mathrm{W}$ \\
\hline$t_{\mathrm{st}}$ & Sleep to idle transient time & $1.162 \mathrm{~ms}$ (measured) & $1.63 \mathrm{~ms}$ \\
\hline$t_{\mathrm{rssi}}$ & RSSI average time & $128 \mu \mathrm{s}$ & 一 \\
\hline$T_{\mathrm{bc}}$ & Beacon cycle period & Varying & - \\
\hline$T_{\text {poll }}$ & Channel polling period & $200 \mathrm{~ms}$ & - \\
\hline$R$ & Data rate & $250 \mathrm{kbps}$ & $1 / 2 \mathrm{Mbps}$ \\
\hline$L_{f}$ & Frame length & 256 bits & 256 bits \\
\hline$N_{\text {nbor }}$ & Number of one-hop neighbors (anchor nodes in radio range) ${ }^{a}$ & 3 & - \\
\hline$N_{\mathrm{lb}}$ & Number of location beacons in one beacon set & 4 & - \\
\hline
\end{tabular}

${ }^{\mathrm{a}}$ Generally, at least three reference points is needed to achieve an unambiguous $2 \mathrm{D}$ location point estimate. Thus, three nodes per maximum transmission coverage area is used as the basis for anchor node density.

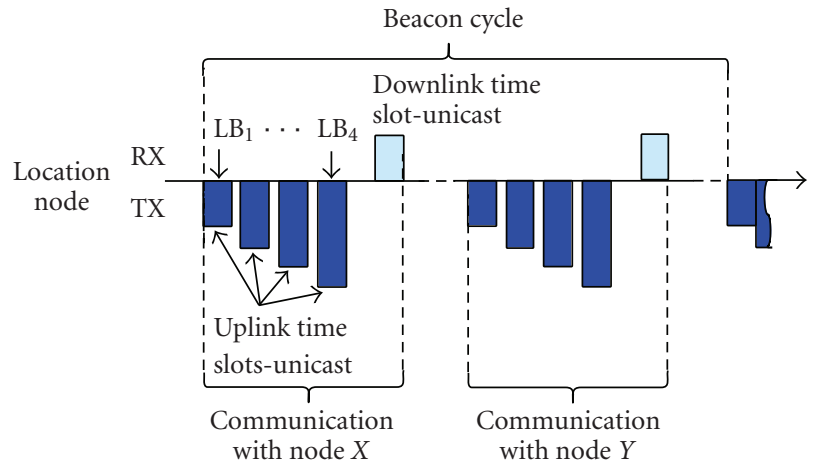

FIGURE 11: Location node beacon cycle using scheduled-MAC-link.

For simplicity and in order to ignore application-specific quantities it is assumed that (1) there is no downlink communication to the location node, but the protocol has to support it, (2) there are neither collisions nor retransmissions, and (3) the clocks are perfectly synchronized, removing the need for reception margins. Furthermore, only radio energy consumption is considered (the energy consumption in WSN nodes is typically dominated by the radio transceiver circuitry [74]).

A frame transmission consists of a radio start-up transient time $\left(t_{\mathrm{st}}\right)$ and the time required by the actual data transmission defined as the ratio of frame length $\left(L_{f}\right)$ and radio data rate $(R)$. During the start-up transient, the power consumption is estimated to be equal to the transmission mode power $P_{\mathrm{tx}(n)}$. Thus, the energy consumption $\left(E_{\mathrm{tx}(n)}\right)$ of a frame transmitted using a power level $n$ is

$$
E_{\mathrm{tx}(n)}=\left(t_{\mathrm{st}}+\frac{L_{f}}{R}\right) P_{\mathrm{tx}(n)}
$$

A frame reception begins with the radio start-up transient and lasts until the frame has been completely received. During a frame reception, the power consumption is equal to the reception mode power $P_{\mathrm{rx}}$. The frame reception energy $\left(E_{\mathrm{rX}}\right)$ is

$$
E_{\mathrm{rx}}=\left(t_{\mathrm{st}}+\frac{L_{f}}{R}\right) P_{\mathrm{rx}}
$$

Since there are no reception margins, both successful and unsuccessful frame receptions consume energy equal to $E_{\mathrm{rx}}$.

Total time required by one carrier sense operation is the sum of a radio start-up transient and the RSSI measurement duration $\left(t_{\mathrm{rssi}}\right)$. Carrier sensing power is equal to the reception-mode power. Thus, the carrier sensing energy $\left(E_{\mathrm{cs}}\right)$ is

$$
E_{\mathrm{cs}}=\left(t_{\mathrm{st}}+t_{\mathrm{rssi}}\right) P_{\mathrm{rx}}
$$

LBs are transmitted $N_{\mathrm{lb}}$ times using power levels ranging from 1 to $N_{\mathrm{lb}}$. Prior to every transmission, contention-MACunsync has to perform CCA, which consumes energy equal to carrier sensing $\left(E_{\mathrm{cs}}\right)$. The rest of the time, the channel is polled at $T_{\text {poll }}$ intervals, each poll consuming energy equal to $E_{\mathrm{cs}}$. Since the transmission of a beacon set consists of $N_{\mathrm{lb}}$ CCA operations, each having duration $\left(t_{\mathrm{st}}+t_{\mathrm{rssi}}\right)$, and LB transmissions, each having duration $\left(t_{\mathrm{st}}+L_{f} / R\right)$, the amount of channel polls $\left(N_{\text {poll }}\right)$ in one beacon cycle $\left(T_{\mathrm{bc}}\right)$ is

$$
N_{\text {poll }}=\left\lfloor\frac{T_{\mathrm{bc}}-N_{\mathrm{lb}}\left(2 t_{\mathrm{st}}+t_{\mathrm{rssi}}+L_{f} / R\right)}{T_{\text {poll }}}\right\rfloor .
$$

The total energy ( $\left.E_{\mathrm{bc} \_ \text {contention_unsync }}\right)$ consumed by contention-MAC-unsync during one beacon cycle is

$$
E_{\text {bc_contention_unsync }}=N_{\mathrm{lb}} E_{\mathrm{cs}}+\sum_{n=1}^{N_{\mathrm{lb}}} E_{\mathrm{tx}(n)}+N_{\text {poll }} E_{\mathrm{cs}} \text {. }
$$


To get the average power consumption $\left(P_{\text {contention_unsync }}\right)$, the total energy ( $\left.E_{\mathrm{bc} \_ \text {contention_unsync }}\right)$ consumed in one beacon cycle is divided by the beacon cycle interval $\left(T_{\mathrm{bc}}\right)$ :

$$
P_{\text {contention_unsync }}=\frac{E_{\text {bc_contention_unsync }}}{T_{\mathrm{bc}}} .
$$

Contention-MAC-sync transmits LBs similarly to contention-MAC-unsync. For downlink traffic, the channel is listened continuously for the duration of the listen period $t_{\text {listen }}$. The listen period length is always the shortest possible. Nodes sending downlink need to perform CCA prior to data transmission. Thus, minimum listen period energy is

$$
E_{\text {listen }}=N_{\text {nbor }}\left(t_{\text {rssi }}+\frac{L_{f}}{R}\right) P_{\mathrm{rx}} \text {. }
$$

The total energy consumption ( $\left.E_{\mathrm{bc} \_ \text {contention_sync }}\right)$ of contention-MAC-sync during one beacon cycle is

$$
E_{\mathrm{bc} \_ \text {contention_sync }}=N_{\mathrm{lb}} E_{\mathrm{cs}}+\sum_{n=1}^{N_{\mathrm{lb}}} E_{\mathrm{tx}(n)}+E_{\text {listen }} .
$$

The average power consumption ( $\left.P_{\text {contention_sync }}\right)$ for contention-MAC-sync is

$$
P_{\text {contention_sync }}=\frac{E_{\text {bc_contention_sync }}}{T_{\mathrm{bc}}} .
$$

In scheduled-MAC-link, the beacon set is transmitted to every neighbor separately. Similarly, downlink slot needs to be received from every neighbor. Thus, the total energy consumption ( $\left.E_{\mathrm{bc} \_s c h e d u l e d \_l i n k}\right)$ during one beacon cycle is

$$
E_{\mathrm{bc} \_s c h e d u l e d \_l i n k}=N_{\mathrm{nbor}}\left(\sum_{n=1}^{N_{\mathrm{lb}}} E_{\mathrm{tx}(n)}+E_{\mathrm{rx}}\right) .
$$

The average power consumption $\left(P_{\text {scheduled_link }}\right)$ for scheduled-MAC-link is

$$
P_{\text {scheduled_link }}=\frac{E_{\text {bc_scheduled_link }}}{T_{\mathrm{bc}}} .
$$

In scheduled-MAC-node, an LB is sent to all neighbors in a single time slot as illustrated in Figure 12. The downlink slot reception amount is still proportional to the neighbor count. The energy consumption ( $\left.E_{\mathrm{bc} \text { _scheduled_node }}\right)$ of scheduled-MAC-node in one beacon cycle is

$$
E_{\mathrm{bc} \_s c h e d u l e d \_n o d e}=\sum_{n=1}^{N_{\mathrm{lb}}} E_{\mathrm{tx}(n)}+N_{\mathrm{nbor}} E_{\mathrm{rx}} \text {. }
$$

The average power consumption ( $\left.P_{\text {scheduled_node }}\right)$ for scheduled-MAC-node is

$$
P_{\text {scheduled_node }}=\frac{E_{\mathrm{bc} \_s c h e d u l e d \_n o d e}}{T_{\mathrm{bc}}} .
$$

The active period of LocMAC base is depicted in Figure 13. Its energy consumption $\left(E_{\mathrm{bc} \_ \text {locmac }}\right)$ is the sum of the energies required by the transmission of the beacon set

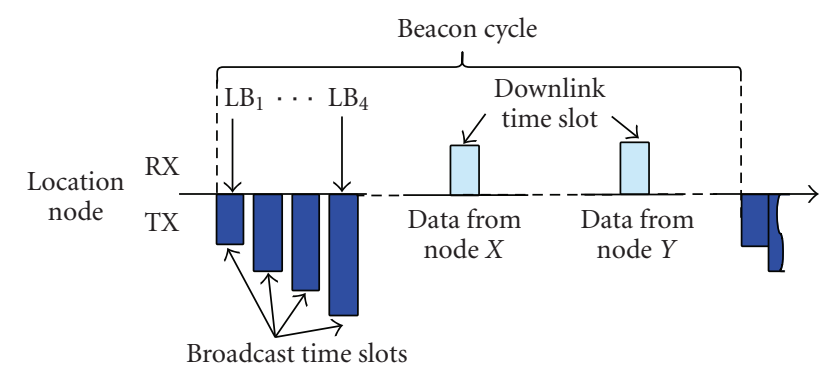

FIgURE 12: Location node beacon cycle using scheduled-MACnode.

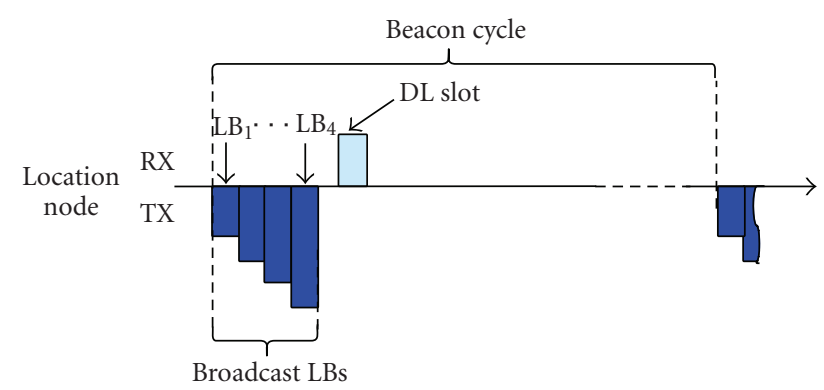

FIGURE 13: LB transmissions with LocMAC.

and the reception of one downlink slot. Thus, the energy consumption of one beacon cycle is

$$
E_{\mathrm{bc} \_ \text {locmac }}=\sum_{n=1}^{N_{\mathrm{lb}}} E_{\mathrm{tx}(n)}+E_{\mathrm{rx}} \text {. }
$$

The corresponding average power consumption $\left(P_{\text {locmac }}\right)$ is

$$
P_{\text {locmac }}=\frac{E_{\mathrm{bc} \_ \text {locmac }}}{T_{\mathrm{bc}}} .
$$

\subsubsection{Neighbor discovery power consumption}

Scheduled contention-access and TDMA-based low dutycycle MAC protocols necessitate a neighbor discovery, when their neighborhood changes. In contrast, low duty-cycle random-access MACs, including LocMAC base, are typically able to broadcast packets without knowledge of their neighbors.

We divide neighbor discovery into initial and maintenance discoveries. An initial neighbor discovery is executed when a node has no known neighbors. This situation can occur at a boot-up, upon entering to the area of an WSN, or due to interference. Maintenance discovery is performed in order to maintain and update connectivity by establishing new links when new neighbors are encountered.

In object localization, both initial and maintenance neighbor discoveries have an essential role. First, the location nodes are not restricted to be in the anchor WSN coverage area all the time. For example, a person accompanied with a location node may carry it outside the network. 
TABLe 2: Additional symbols, descriptions, and defined values for neighbor discovery power consumption analysis.

\begin{tabular}{lll}
\hline Symbol & Description & Value \\
\hline$r$ & $\begin{array}{l}\text { Maximum radio range } \\
\text { Range of sufficient signal strength com- } \\
\text { pared to maximum radio range with }\end{array}$ & 0.5 \\
$\rho$ & ENDP & \\
& Cluster beacon transmission rate & $0.5 \mathrm{~Hz}$ \\
$f_{\mathrm{cb}}$ & Network beacon transmission rate & $0.5 \mathrm{~Hz}$ \\
$f_{\mathrm{nb}}$ & $\begin{array}{l}\text { Network beacon reception rate } \\
f_{\mathrm{nbrx}}\end{array}$ & $0.5 \mathrm{~Hz}$ \\
$k$ & $\begin{array}{l}\text { The amount of neighbors to which } \\
\text { synchronization is maintained (equal } \\
\left.N_{\text {nbor }}\right)\end{array}$ & \\
\hline
\end{tabular}

This kind of scenarios can result in large amounts of redundant initial neighbor discovery attempts, and high energy consumption while being offline. To conserve energy, the neighbor discovery period could be gradually increased after each initial neighbor discovery attempt. Yet, this would increase the latency of finding neighbors, when (re)entering the anchor WSN coverage area.

Secondly, location nodes can be highly mobile introducing dynamics in the network. This sets stress on the maintenance neighbor discovery protocol. Thus, it can present significant energy overhead during online phase.

Next, we will derive models for neighbor discovery power consumption. Conventional network scanning is considered first. Then, neighbor discovery using ENDP follows. The analysis utilizes symbols given in Table 1 and additional symbols given in Table 2 .

To make network scanning more energy-efficient, a network beacon signaling scheme introduced in [21] is used. A network-wide fixed channel is used to transmit network beacons containing node status information for the selection of an appropriate neighbor for association. The transmission of frequent network beacons reduces the energy consumption in dynamic networks significantly [58]. The beacons used for link establishment are referred to as cluster beacons (the terminology used in [21] is adopted. Cluster beacons do not necessarily imply the usage of clustered network topology). They contain information that is vital for data exchanges.

When a node moves out of its neighbors radio range $(r)$, a link failure occurs. For a node moving at speed $v$ and having links to $N_{\text {nbor }}$ nodes, the link failure rate $\left(f_{\mathrm{lf}}\right)$ can be approximated to be [21]

$$
f_{\mathrm{lf}}=\frac{N_{\mathrm{nbor}} v}{r}
$$

Nodes transmit network beacons with rate $f_{\mathrm{nb}}$. For discovering all possible neighbors inside its radio range, a node needs to listen to the network channel for a whole network beacon period. Thus, the network scan duration is

$$
t_{\mathrm{ns}}=\frac{1}{f_{\mathrm{nb}}} .
$$

In order to continuously maintain sufficient amount of links, a mobile node needs to discover new neighbors at least every time a link is lost. Thus, using network scanning as neighbor discovery method, the scan interval $\left(T_{\mathrm{ns}}\right)$ is

$$
T_{\mathrm{ns}}=\frac{1}{f_{\mathrm{lf}}} .
$$

The long-time average power consumed by network scanning in maintenance discovery is

$$
P_{\text {ns_maintenance }}=\frac{\left(t_{\mathrm{st}}+t_{\mathrm{ns}}\right) P_{\mathrm{rx}}}{T_{\mathrm{ns}}} .
$$

Next, analytical expressions for neighbor discovery power consumption using ENDP are derived. To save space, a detailed derivation of the expressions is omitted. For more in-depth description of the derivation and equations, we refer the readers to [21].

ENDP can maintain links with proactive node schedule distribution as long as it receives valid synchronization data units (SDUs) from its $k$ neighbors. After a link failure, a node tries to synchronize to neighbors using information provided by the SDUs. Synchronization is attempted until an attempt is successful, or all $\left(k^{2}\right)$ SDUs are examined. An invalid SDU contains data about a node that is out of the radio range making synchronization to it impossible.

Assuming uniform node distribution, the probability $(q)$ that the received $k^{2}$ SDUs from $k$ neighbors are invalid and a network scan is required is

$$
q=\prod_{a=1}^{k}\left(1-\frac{N_{\text {nbor }} p_{\text {valid }}}{N_{\text {nbor }}-(a-1)}\right)^{k},
$$

where $p_{\text {valid }}(\approx 59 \%)$ is the probability that the received SDU is valid and $a$ denotes the SDU index.

The probability of finding a neighbor in the first attempt

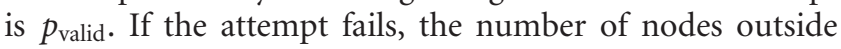
the radio range decreases. The expected number of network beacon receptions $(u)$ until a neighbor inside the radio range is found is

$$
\begin{aligned}
u= & p_{\text {valid }}+\sum_{a=2}^{k^{2}-1}\left(a \prod_{b=1}^{a-1}\left(1-\frac{N_{\text {nbor }} p_{\text {valid }}}{N_{\text {nbor }}-(b-1)}\right) \frac{N_{\text {nbor }} p_{\text {valid }}}{N_{\text {nbor }}-(a-1)}\right) \\
& +k^{2} \prod_{a=1}^{k^{2}-1}\left(1-\frac{N_{\text {nbor }} p_{\text {valid }}}{N_{\text {nbor }}-(a-1)}\right) .
\end{aligned}
$$

When having no neighbors, a node needs to scan until a network beacon with sufficient signal strength is received. The range of sufficient signal strength in proportion to maximum radio range $(r)$ is defined to be $\rho$. Similarly to (29), the expected number of network beacon receptions $\left(N_{\mathrm{nb}}\right)$ until a beacon with sufficient signal strength is received is

$$
\begin{aligned}
N_{\mathrm{nb}}= & \rho^{2}+\sum_{a=2}^{N_{\text {nbor }}-1}\left(a \prod_{b=1}^{a-1}\left(1-\frac{N_{\text {nbor }} \rho^{2}}{N_{\text {nbor }}-(b-1)}\right) \frac{N_{\text {nbor }} \rho^{2}}{N_{\text {nbor }}-(a-1)}\right) \\
& +N_{\text {nbor }} \prod_{a=1}^{N_{\text {nbor }}-1}\left(1-\frac{N_{\text {nbor }} \rho^{2}}{N_{\text {nbor }}-(a-1)}\right) .
\end{aligned}
$$


The required network scan duration $\left(t_{\text {ns_endp }}\right)$ can be derived to be

$$
t_{\text {ns_endp }}=\frac{N_{\mathrm{nb}}}{f_{\mathrm{nb}} N_{\mathrm{nbor}}} .
$$

The long-time average power of network scans $\left(P_{\text {ns_endp }}\right)$ using ENDP is

$$
P_{\text {ns_endp }}=f_{\mathrm{lf}} q\left(t_{\mathrm{st}}+t_{\mathrm{ns \_ endp}}\right) P_{\mathrm{rx}} \text {. }
$$

To enable neighbor discovery and synchronization, a node transmits network and cluster beacons at rates $f_{\mathrm{nb}}$ and $f_{\mathrm{cb}}$, respectively. Cluster beacons are received at rate $f_{\mathrm{cb}}$ from the $k$ neighbors to which synchronization is maintained. Network beacons piggybacking SDUs are received at rate $f_{\text {nbrx }}$ from the same $k$ neighbors. After every link failure, which occurs at rate $f_{\mathrm{lf}}$, on average $u$ cluster beacon reception attempts are needed when exploiting SDU information. Thus, the average power consumed by the beacon exchanges is

$$
P_{b}=\left(f_{\mathrm{nb}}+f_{\mathrm{cb}}\right) E_{\mathrm{tx}}+k\left(f_{\mathrm{cb}}+f_{\mathrm{nbrx}}\right) E_{\mathrm{rx}}+f_{\mathrm{lf}}\left((u-1) E_{\mathrm{rx}}+E_{\mathrm{rx}}\right) .
$$

ENDP maintenance discovery power consists of the network scan power $\left(P_{\text {ns_endp }}\right)$ and the beacon exchange power $\left(P_{b}\right)$. The total ENDP maintenance power $\left(P_{\text {endp_maintenance }}\right)$ is

$$
P_{\text {endp_maintenance }}=P_{\text {ns_endp }}+P_{b} \text {. }
$$

\subsubsection{The models and their relation to real protocols}

The described ideal protocol models achieve somewhat better performance than the real protocols they represent. However, the goal of the analysis is to dissect and validate the usage of an application-specific MAC protocol (LocMAC) in localization. The mutual performance of the individual WSN MAC protocols in question is not under examination. Thus, the usage of a generalizing protocol models is justified as long as they do not perform worse than any of the represented real protocols.

Contention-MAC-unsync represents low duty-cycle random-access MAC protocols, namely WiseMAC, B-MAC, SpeckMAC, X-MAC, and SCP-MAC. In reality, the energy consumptions of WiseMAC, B-MAC, SpeckMAC, and $\mathrm{X}$-MAC, will be bigger due to extended preambles (length varies with protocol), and overhearing. Also, if there is downlink data, the preambles will add even more costs. SCPMAC power consumption will be increased by contention windows, wake-up tone transmissions, and overhearing.

Contention-MAC-sync represents scheduled contentionaccess MAC protocols, such as S-MAC, T-MAC, and IEEE 802.15.4. The power consumption of the actual protocols will be increased due to relatively long listen periods causing idle listening and overhearing. In contention-MAC-sync, the listen period is modeled to be only as long as is needed for successful communication.

The power consumption of LEACH, SMACS, and TUTWSN MAC represented by scheduled-MAC-link, and

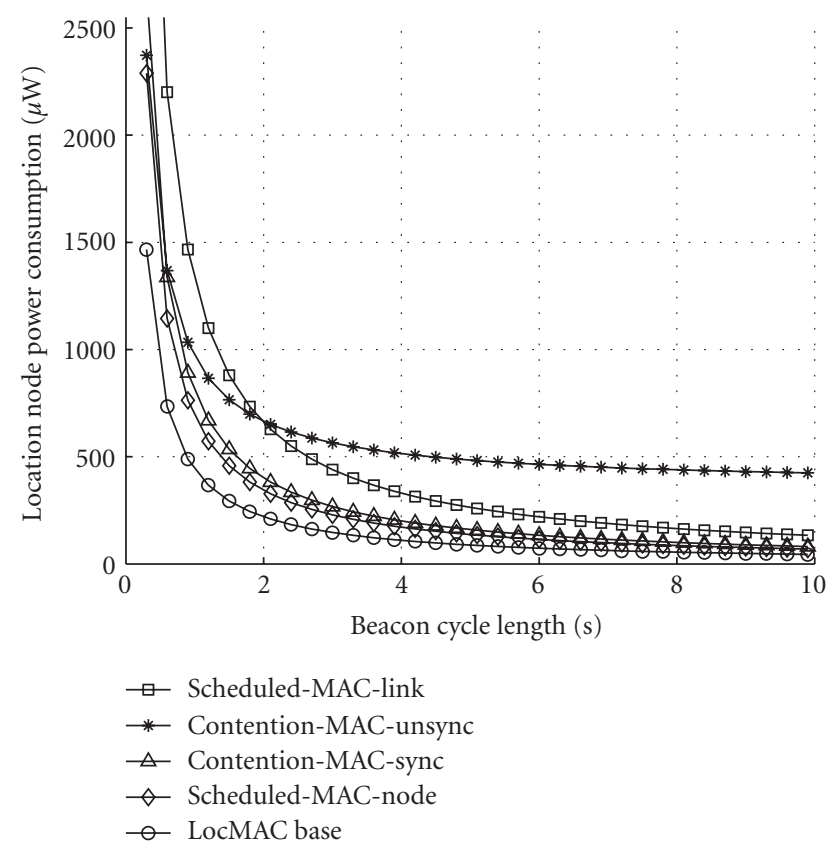

FIgUre 14: Power consumption of scheduled-MAC-link, contention-MAC-unsync, contention-MAC-sync, scheduled-MAC-node, and LocMAC base as a function of beacon cycle length with static location node.

PACT and TRAMA represented by scheduled-MAC-node will increase as the number of anchor nodes in their radio range increases.

For the protocols needing synchronization (all scheduled contention-access and TDMA protocols, and SCP-MAC), the situation represented by the protocol models occurs only in fully static and ideal conditions with no link breakages. If the location node is mobile, neighbor discovery power consumption has to be added. Also, synchronization inaccuracy will increase the power consumption.

For the low duty-cycle random-access protocols, other than SCP-MAC, contention-MAC-unsync applies both in static and in mobile scenarios.

\subsubsection{Power consumption comparison}

For power consumption comparison, the values given in Tables 1 and 2 are used. Chipcon CC2420 includes RSSI functionality. Thus, it supports all the discussed protocols and is used to derive power consumption values for all of them. The values for nRF24L01 are given for comparison. As can be seen, nRF24L01 consumes less power than CC2420. The data rate of nRF24L01 is higher than the data rate of CC2420. This results in frames with shorter duration, which further decreases the energy consumption of transmitted and received frames.

Figure 14 illustrates and clarifies the power consumption of scheduled-MAC-link, contention-MAC-unsync, contention-MAC-sync, scheduled-MAC-node, and LocMAC base as a function of beacon cycle length $\left(T_{\mathrm{bc}}\right)$ in a static location node case. LocMAC base outperforms the ideal protocol 


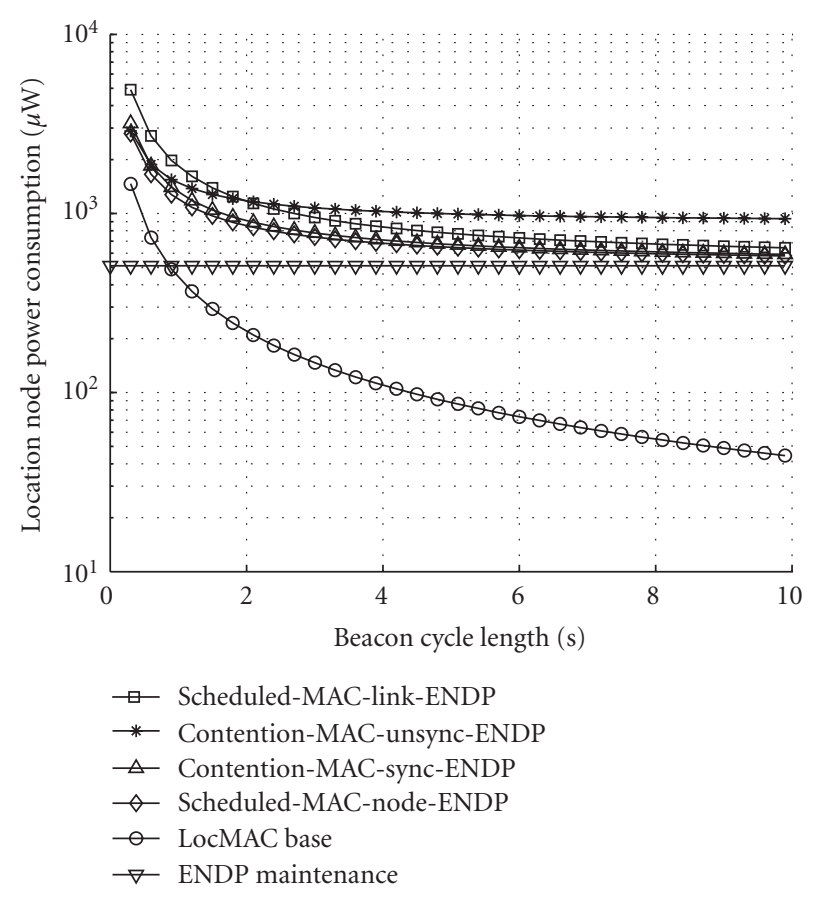

(a) Power consumption when location speed is $1 \mathrm{~m} / \mathrm{s}$

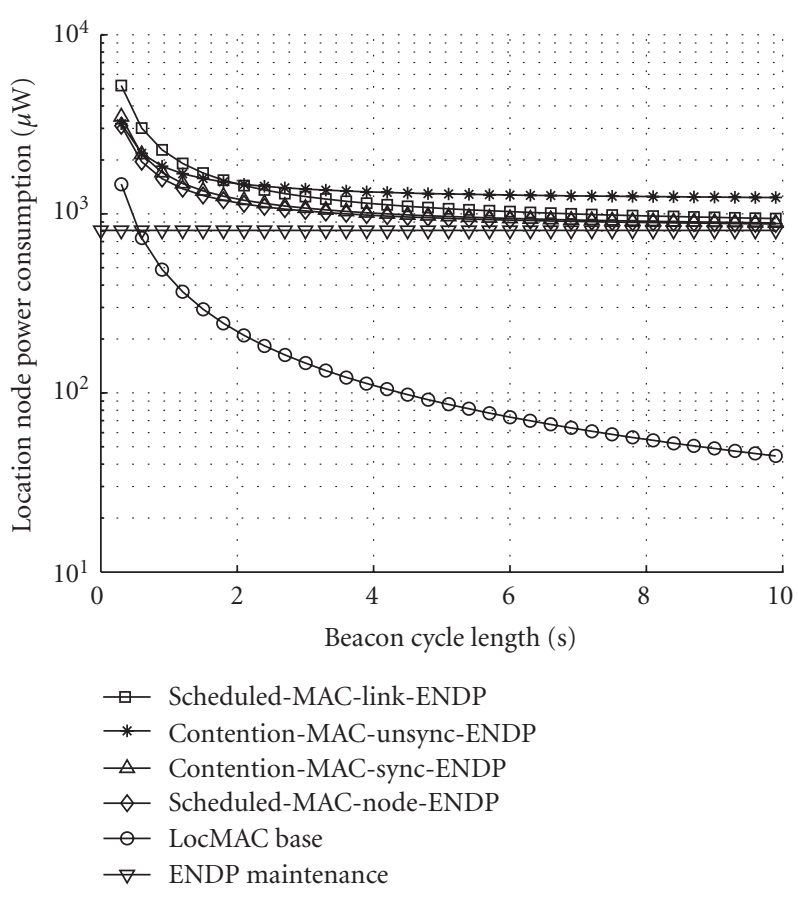

(b) Power consumption when location speed is $3 \mathrm{~m} / \mathrm{s}$

Figure 15: Power consumptions of scheduled-MAC-link, contention-MAC-unsync, contention-MAC-sync, scheduled-MAC-node, and LocMAC base as a function of beacon cycle length when location node is mobile and ENDP is used as the neighbor discovery method.

models independent of the beacon cycle length. For example, at 1 -second beacon cycle LocMAC base consumes 1.5 to 3 times less power than the protocol models. With 10 second beacon cycle, the power consumption is 1.5 to 9.5 times lower. As the beacon cycle length increases, and location update rate decreases, the power consumption of the scheduled protocols starts to approach the power consumption values of LocMAC base. This happens because sleep mode power consumption starts to dominate as the duty cycle decreases. With contention-MAC-unsync, the idle listening power consumption caused by channel polling becomes more dominant.

When network scanning is used as the neighbor discovery method, its power clearly dominates the power consumption of the protocol models. The network scanning power consumption alone is 69 times LocMAC base power with 1-second beacon cycle and 761 times LocMAC base power with 10-second beacon cycle when the location node speed is $1 \mathrm{~m} / \mathrm{s}$. Corresponding values are 207 and 2285, respectively, when the location node speed is $3 \mathrm{~m} / \mathrm{s}$.

Figures 15(a) and 15(b) depict power consumptions with mobile location node moving at speeds $1 \mathrm{~m} / \mathrm{s}$ and $3 \mathrm{~m} / \mathrm{s}$, respectively, when ENDP is used as the neighbor discovery method. With 1-m/s location node speed and 1-second beacon cycle, LocMAC base performs from 2.5 to 4 times better than the protocol models. When the beacon cycle takes 10 seconds, 13-21 times smaller power consumption is achieved. LocMAC base performs approximately from 3 to

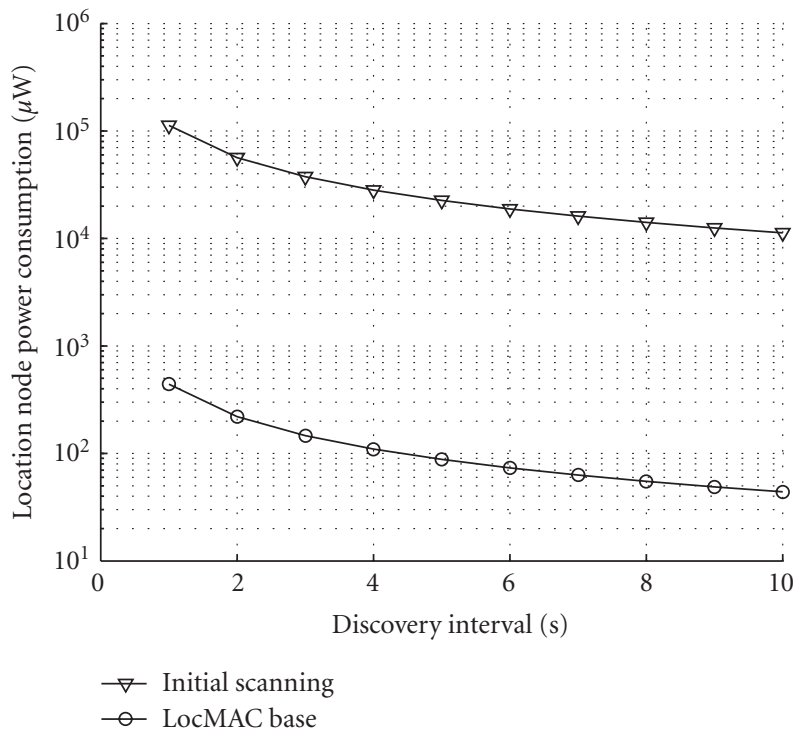

FIgURE 16: Power consumptions of LocMAC and initial scanning as a function discovery interval (i.e., beacon cycle length for locMAC and scan interval for scanning).

5 times better, when the location node speed is $3 \mathrm{~m} / \mathrm{s}$ and 1 -second beacon cycle is used. At 10-second beacon cycle, from 20 to 28 times better power consumption values are obtained. 
Figure 16 illustrates initial network scanning power consumption as a function of the scan interval. LocMAC base consumes approximately 256 times less power than initial scanning.

\subsection{Location node collision avoidance evaluation}

To evaluate the effectiveness of LocMAC base, the APSR CA method is simulated in Matlab. The simulations measure the amount of APSR rounds needed to achieve completely conflict-free schedule having no colliding active period slots as a function of location node amount situated in the same radio coverage area. The results are given as an average and maximum values of 1000 simulation runs.

Using the values given for nRF24L01 in Table 1 and $1 \mathrm{Mbps}$ data rate results in 18.86-milliseconds long active period slot. The beacon cycle length is set to be 2 seconds. This gives a total of 106 active period slots.

Figure 17(a) presents the amount of APSR rounds needed to achieve schedules with no overlapping slots. In the average case, when the location node amount is around 55 (approximately half of the total APSR slot amount) the amount of needed APSR rounds starts to increase fast. In the worst case (Max), the curve follows the same trend as the average curve, but with approximately five-fold increase in APSR rounds.

Figure 17(b) depicts the the maximum amount of conflicts a location node encountered before the schedule was totally collision-free. For example, with 55 location nodes the total APSR round amount is already around 20 (average, Figure $17(\mathrm{a})$ ), but from these rounds a specific location node is at worst involved in 8 . The worst case values (Max) show approximately three-fold increase to the average case.

Figure 17 (c) illustrates the maximum number of consecutive conflicting slots that a location node encountered in the simulations. For example, with 55 location nodes the average value is around two, meaning that localization data update still happens at latest after two APSR rounds. Corresponding maximum values show that two consecutive conflicting APSR rounds for a location node can happen already with six location nodes. Fortunately, the increase in worst case consecutive conflicts is relatively small as location node amount increases.

Similar simulations with 4-second beacon cycle show that the point after which APSR rounds start to increase fast is approximately around 110 location nodes. This is double the amount of location nodes with which the same effect happened with 2-second beacon cycle. This result indicates that LocMAC congestion adaptation works effectively.

\section{LOCALIZATION EXPERIMENTS, RESULTS, AND EVALUATION}

The feasibility of our localization approach is evaluated using real WSN prototype nodes communicating in the $2.4 \mathrm{GHz}$ frequency band. Different experiment scenarios and multiple metrics are used to obtain thorough knowledge about the operation.

\subsection{Prototype platforms}

The anchor and location node prototypes are presented in Figures 18(a) and 18(b), respectively. The anchor node prototype platform uses a Microchip PIC18F8722 microcontroller unit (MCU), which integrates an 8-bit processor core with $128 \mathrm{kB}$ of FLASH program memory, $4 \mathrm{kB}$ of RAM data memory, and $1 \mathrm{kB}$ EEPROM. The location node platform uses a microchip PIC18F2520 MCU, which integrates an 8bit processor core with $32 \mathrm{kB}$ of FLASH program memory, $1536 \mathrm{~B}$ of RAM data memory, and 256 B EEPROM. The used clock speed for both discussed MCUs is $8 \mathrm{MHz}$ resulting in 2 MIPS performance.

For wireless communication, both prototype platforms use a Nordic semiconductor nRF24L01 $2.4 \mathrm{GHz}$ radio transceiver having data rate of $1 / 2 \mathrm{Mbps}$ and $80 / 40$ available frequency channels in the industrial, scientific, and medical (ISM) unlicensed radio band. Transmission power level is selectable from four power levels between $-18 \mathrm{dBm}$ and $0 \mathrm{dBm}$ with $6 \mathrm{dBm}$ intervals and $\pm 4 \mathrm{dBm}$ accuracy.

Both prototype platforms include a Maxim DS620 temperature sensor and a VTI Technologies SCA3000-E02 3 -axis accelerometer. In addition, the anchor node platform has an Avago Technologies APDS-9002 ambient light photo sensor for illuminance measurements.

To enable anchor node usage as a gateway, it can be equipped with a Lantronix XPort embedded Ethernet device server, which bridges the MCU serial port to Ethernet.

\subsection{Experiments}

The experiments were performed with two test scenarios both deployed at Department of Computer Systems (DCSs) at TUT. The environment includes typical office rooms. Most of the walls are wooden, but also few glass and steel walls exist. During the experiments, the TUT WLAN network was active producing interference to our localization network communication. Both scenarios covered the same floor area of approximately $700 \mathrm{~m}^{2}$, but with a differing amount of anchor nodes. Scenario 1, depicted in Figure 19(a), consisted of 23 anchor nodes deployed in 21 rooms, one anchor node per room, and two in the hallway. Scenario 2 included 12 anchor nodes placed in 12 rooms, approximately one anchor node in every other room. The anchor nodes were relatively evenly distributed in the covered area.

The used test locations for both scenarios are depicted in Figure 20. In Scenario 1, a total of 35 test locations was recorded. The test locations included at least one test location per room and six test locations in the hallway. In two of the rooms, four additional test locations per room were used in order to find out how small changes in real locations affected the localization result. Scenario 2 had 27 test locations, including one test location per room (total of 21 test locations) and six test locations in the hallway.

\subsection{Results and evaluation}

Results are derived using several evaluation metrics in order to evaluate the localization performance thoroughly and in order to find out the most applicable way of using the design. 


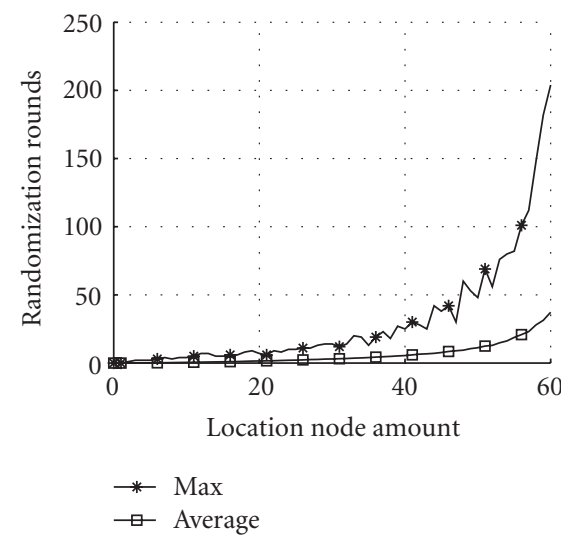

(a) Needed APSR rounds to achieve schedules with no overlapping slots as a function of location node amount

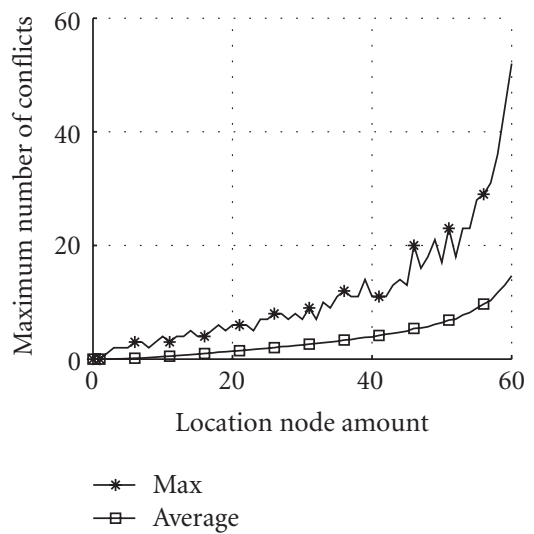

(b) Maximum number of conflicts for individual location node as a function of location node amount

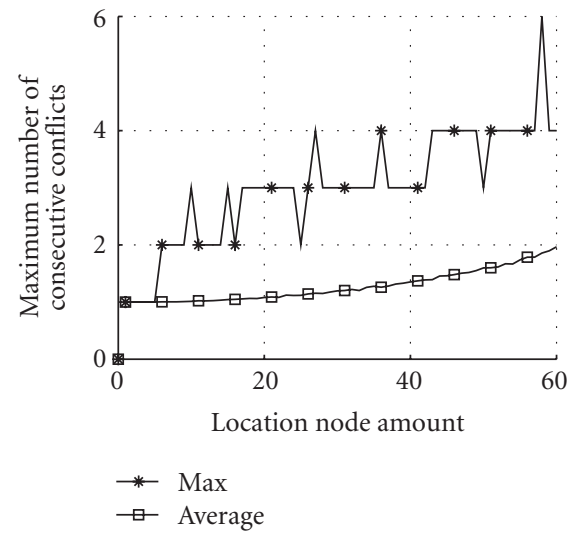

(c) Maximum number of consecutive conflicts for individual location node as a function of location node amount

FIGURE 17: Active period slot randomization procedure performance with differing amount of location nodes, 2-second beacon cycle.

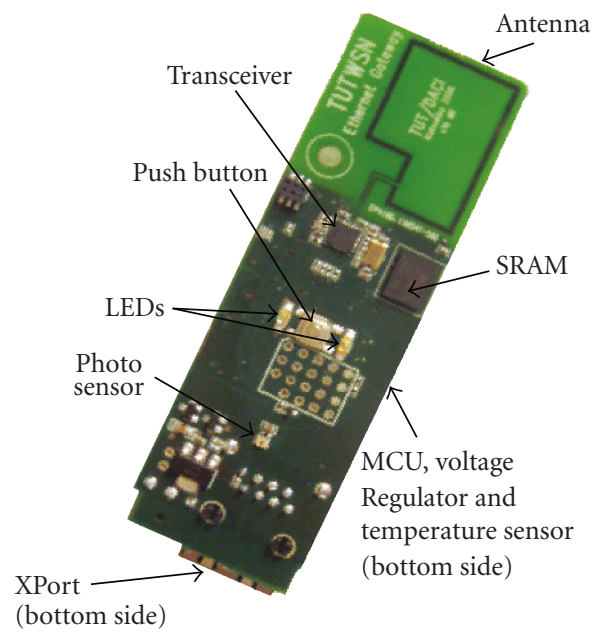

(a) Anchor node prototype

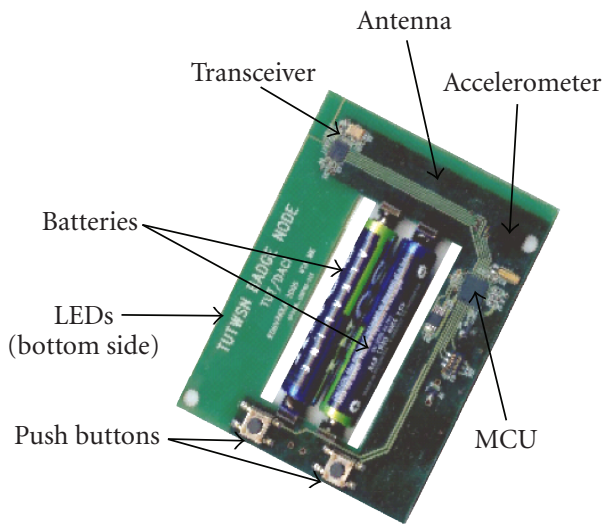

(b) Location node prototype

FIGURE 18: Anchor node and location node prototype platforms.

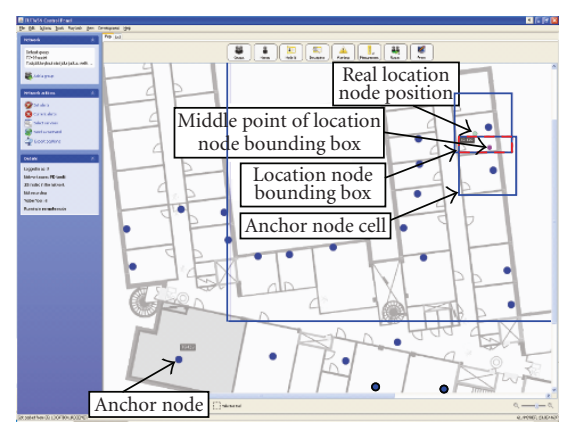

(a) Scenario 1 in monitoring user interface

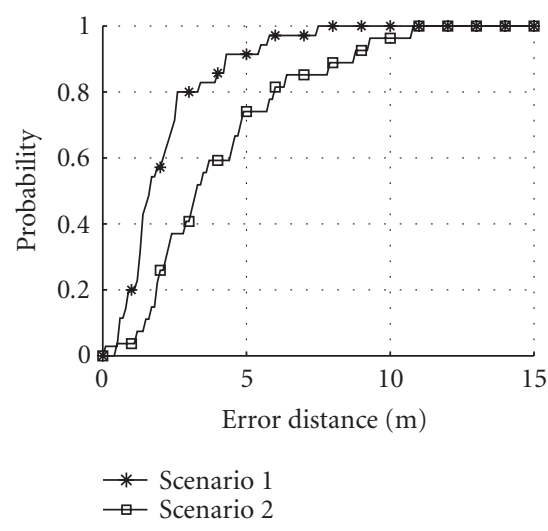

(b) Point based accuracy CDFs

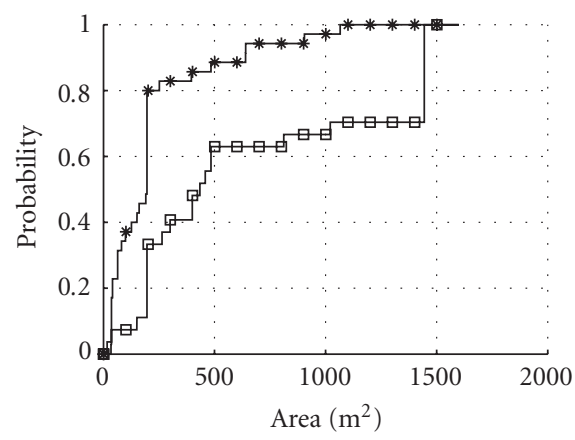

$*$ Scenario 1

๑- Scenario 2

(c) Bounding box accuracy CDFs

FIGURE 19: Localization test scenario 1 in user interface and cumulative distribution function results for point-based and bounding box accuracies in Scenario 1 (23 anchor nodes) and Scenario 2 (12 anchor nodes). 


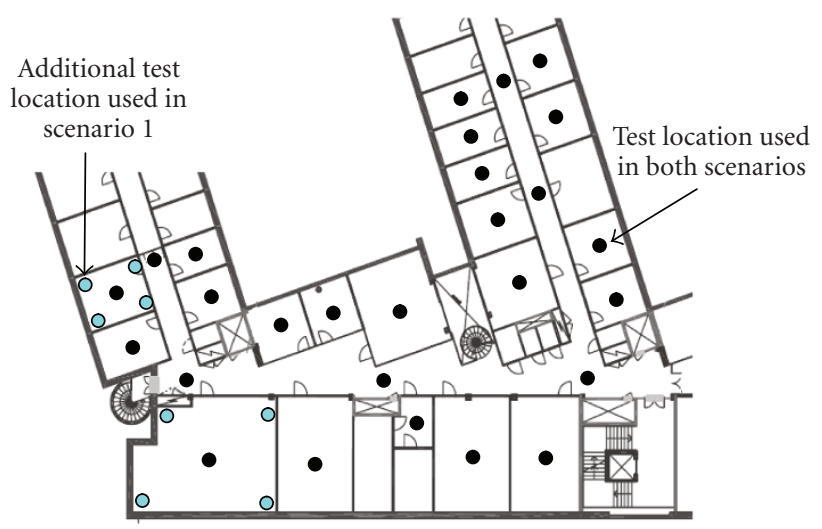

Figure 20: Test locations used in the experiments. Both scenarios included one test location per room and six test locations in the hallway (solid dots). For scenario 1, four additional test locations (open dots) in two rooms were recorded. This was done in order to find out the localization granularity by experimenting how small changes in real locations affected the localization result.

\subsubsection{Point-based evaluation}

To enable comparison with previous localization solutions, an absolute Euclidean error distance metric is used. Choosing the bounding box middle point as the location estimate minimizes the expected value of the possible error distance if the real location can be in any point in the bounding box area. Thus, point base accuracy is evaluated as the Euclidean distance from estimated bounding box middle point to the real node location. Precision is given by the cumulative distribution function (CDF) comprised of all the accuracy values.

To evaluate the localization performance according to point-based metrics, an absolute Euclidean distance between the real location and the estimated location was calculated for every test point. The resulting CDFs for Scenario 1 and Scenario 2 are illustrated in Figure 19(b). In Scenario 1, $25 \%$ of the estimated locations are within $1.3 \mathrm{~m}$ of the real location, $50 \%$ within $1.7 \mathrm{~m}, 75 \%$ within $2.6 \mathrm{~m}$, and $90 \%$ within $4.3 \mathrm{~m}$. For Scenario 2, the corresponding 25th, 50th, 75 th, and 90th percentile values are $2.0 \mathrm{~m}, 3.3 \mathrm{~m}, 5.8 \mathrm{~m}$, and $8.8 \mathrm{~m}$, respectively.

\subsubsection{Area-based evaluation}

To fairly evaluate the bounding box algorithm, we need to address bounding box accuracy and precision. We define bounding box accuracy to be the area of the bounding box. Bounding box precision is the ability to estimate bounding boxes correctly. It is given as the percentage of times the returned bounding box actually contains the true location node location.

The CDFs for bounding box accuracies are depicted in Figure 19(c). In Scenario 1, the 25th, 50th, 75th, and 90th percentile bounding box accuracies are $64 \mathrm{~m}^{2}, 196 \mathrm{~m}^{2}$, $196 \mathrm{~m}^{2}$, and $640 \mathrm{~m}^{2}$, respectively. All the test locations fall into the corresponding resolved bounding box area giving a $100 \%$ precision. In Scenario 2, 25th, 50th, 75th, and 90th percentile bounding box accuracies are $196 \mathrm{~m}^{2}, 434 \mathrm{~m}^{2}$, $1444 \mathrm{~m}^{2}$, and $1444 \mathrm{~m}^{2}$, respectively. Scenario 2 results in $96.3 \%$ of the real locations being in the resolved bounding box area.

The bounding boxes in the experiments are relatively large. This is due to the fact that a rather conservative radio propagation model leading to long radio ranges was used. This leads to better bounding box precision at the cost of accuracy.

To make the bounding boxes smaller, the radio propagation model should be tuned. In environments with lots of small rooms, signals with higher transmission powers will have to penetrate more walls. This indicates that increasing the path loss exponent value would give more realistic transmission power to distance mappings. At the same time, the iterative bounding boxes algorithm would be given more weight in the location estimation process.

Furthermore, areas outside the building could be removed from the bounding boxes. However, this would require more complex processing and that the location resolution algorithm would have specific knowledge of the floorplan map. The solution is out of the scope of this paper.

\subsubsection{Room-level evaluation}

Room-level precision is given as a percentage of times the location node room is determined correctly.

When analyzing the experiment data, it was noted that the middle point of the bounding box correlated with the room the real location was situated in very well. When using bounding box middle point as the room determination criteria, the room was estimated correctly $89.7 \%$ of times in Scenario 1 and $52.4 \%$ of times in Scenario 2. In rooms including an anchor node, the location node bounding box was usually centered correctly. This results from the small cell given by the anchor node in the same room receiving LBs without a lot of obstructions. The main cause of room determination performance deterioration when changing from Scenario 1 to Scenario 2 is the lack of anchor nodes inside the same room as the location node leading to bigger cell sizes and more coarse-grained bounding boxes.

\subsubsection{Robustness evaluation}

Lorincz and Welsh [32] require a localization system to have no single point of failure and a localization algorithm that can gracefully handle incomplete data and failed nodes in order for it to be robust. We acknowledge that our system, as it is currently implemented, has one possible single point of failure; the location resolver situated in a server. However, the server can be placed in a secure location and maintained, significantly reducing its possibility for failure and hastening recovery if a failure should occur. Furthermore, this single point of failure can be removed by decentralizing the location estimation to the network as will be discussed later.

The localization system robustness against anchor node failure and incomplete data was analyzed comparing the localization performance of the two test scenarios, Scenario 


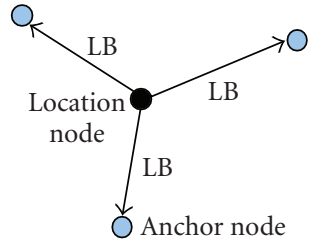

(a) Location node transmits LBs

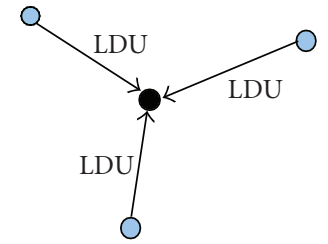

(b) Anchor nodes communicate heard LB data, their own coordinates, and local transmission power to range mapping values to the location node in an LDU frame

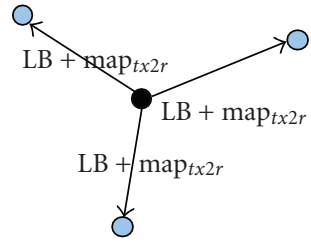

(c) Location node has resolved new transmission power to range mapping values and communicates them to anchor nodes by piggybacking the data in LBs

FIGURE 21: Distributed localization.

TABLE 3: Comparison of point-based and bounding box accuracies for Scenario 1 and Scenario 2.

\begin{tabular}{|c|c|c|c|c|}
\hline \multirow{3}{*}{ Percentile } & \multicolumn{4}{|c|}{ Point-based accuracy [m] } \\
\hline & \multicolumn{2}{|c|}{ Scenario 1} & \multicolumn{2}{|c|}{ Scenario 2} \\
\hline & Original & Normalized & Original & Normalized \\
\hline 25 th & 1.3 & 1.3 & 1.9 & 1 \\
\hline 50 th & 1.7 & 1.7 & 3.2 & 1.7 \\
\hline 75 th & 2.6 & 2.6 & 5.8 & 3 \\
\hline 90th & 4.3 & 4.3 & 8.8 & 4.6 \\
\hline \multirow{3}{*}{ Percentile } & \multicolumn{4}{|c|}{ Bounding box accuracy $[\mathrm{m}]$} \\
\hline & \multicolumn{2}{|c|}{ Scenario 1} & \multicolumn{2}{|c|}{ Scenario 2} \\
\hline & Original & Normalized & Original & Normalized \\
\hline 25 th & 64 & 64 & 196 & 102 \\
\hline 50 th & 196 & 196 & 433 & 226 \\
\hline 75 th & 196 & 196 & 1443 & 753 \\
\hline 90th & 640 & 640 & 1443 & 753 \\
\hline
\end{tabular}

2 having approximately half the anchor node amount of Scenario 1.

In order to make qualitative comparison between the two test scenarios, the point-based and bounding box accuracy values of Scenario 2 are normalized to the case of 23 anchor nodes. That is the values are multiplied by 12 (anchor nodes) $/ 23$ (anchor nodes). The original and normalized values are presented in Table 3 .

As can be observed from Table 3, the normalized pointbased accuracy values show a similar trend, but in Scenario 2 the accuracy deteriorates slightly faster than in Scenario 1. This can also be determined by comparing the shapes of the curves in Figure 19(b).

Area-based performance results indicate that precision decreased only by 3.7 percentage units when the anchor node amount was halved. However, the bounding box values in Table 3 and the curve shapes in Figure 19(c) show that with Scenario 1 the majority of the accuracy values are at the smaller end of the scale, whereas Scenario 2 produces more steady bounding box accuracy value deterioration.
The ability to infer correct room decreased from $89.7 \%$ to $52.4 \%$ when changing from Scenario 1 to Scenario 2 . This means that room-level precision decreased by $41.6 \%$ as anchor node amount was reduced by $47.8 \%$.

The comparison of the two test scenarios shows that the localization performance correlates with the amount of anchor nodes. The performance degradation is more or less linearly dependent on the anchor node amount. Thus, it can be considered to be graceful. As long as there is radio coverage over the localized area, the presented scheme shows robustness against anchor node failure and incomplete data.

\subsubsection{Granularity}

To test the localization granularity limits of the presented localization scheme, four additional test locations (Figure 20) for two rooms were recorded in Scenario 1. The locations estimated in five different points in a room did not show increased correlation to their real locations inside the room. This results from the fact that small changes in real locations rarely break radio coverage boundaries for adjacent transmission power levels.

The measurements indicate that room-level granularity approaches the limit reached with the current implementation. Hence, adding more anchor nodes than one per room would result in diminishing returns. To reach smaller granularity, the radio ranges should be decreased and a denser anchor network deployed, and/or it should be possible to use more radio transmission power levels.

\section{COMPARISON AGAINST RELATED LOCALIZATION PROPOSALS}

Next, our localization approach will be compared against other RF-based solutions. The discussion is divided into applicability issues and location estimation performance comparison. The applicability of a solution is mainly dictated by the indirect costs it inflicts to enable localization. Location estimation performance comparison discusses the load imposed by different location estimation algorithms and the accuracy achieved by different approaches. 
TABLE 4: Costs of compared localization approaches.

\begin{tabular}{|c|c|c|c|c|c|}
\hline \multirow{2}{*}{ Proposal } & \multirow{2}{*}{$\begin{array}{c}\text { Our approach } \\
\text { (WSN) }\end{array}$} & \multicolumn{2}{|c|}{ Rंange- and proximity-based } & \multicolumn{2}{|c|}{ Scene analysis } \\
\hline & & WSN & WLAN & WSN & WLAN \\
\hline \multicolumn{6}{|l|}{ Time costs } \\
\hline Autonomous network formation and maintenance & Yes & Yes & No & Yes & No \\
\hline Network setup time & Low & Low & Medium & Medium & High \\
\hline Location node battery change interval & Longest & Short to medium & Short & Short to medium & Short \\
\hline Anchor network battery change interval & Long to infinite & Medium & Infinite & Medium & Infinite \\
\hline Composite & Lowest & Low & Medium & High & Highest \\
\hline \multicolumn{6}{|l|}{ Space Costs } \\
\hline Location node size & Lowest & Lowest to low & Medium & Low & Medium \\
\hline Anchor node size & Lowest & Lowest to low & High & Low & High \\
\hline Anchor node density & Medium & Medium & Low & Medium & Low \\
\hline Other infrastructure space cost & No & No & Yes & No & Yes \\
\hline Composite & Lowest & Lowest to low & Medium & Low & Medium \\
\hline \multicolumn{6}{|l|}{ Capital Costs } \\
\hline Location node unit cost & Lowest & Lowest to low & Medium & Low & Medium \\
\hline Anchor node unit cost & Lowest & Lowest to low & High & Low & High \\
\hline Other infrastructure costs & No & No & Yes & No & Yes \\
\hline Anchor network aggregate cost & Low & Lowest to low & Medium & Low & Medium \\
\hline Composite & Lowest & Lowest to low & Medium & Low & Medium \\
\hline
\end{tabular}

\subsection{Applicability}

Localization network costs can be divided into time, space, and capital costs [23]. Time costs comprise of the installation procedure time consumption, and the maintenance and administration costs of the network. The amount of installed infrastructure and the size and form factor of the used HW make up space costs. Capital costs include the location node and anchor node unit costs. Furthermore, other infrastructure costs and the salaries of the installation and support personnel can be added up to the capital costs. We do not include salaries to the discussion since they are highly scenario-specific.

The costs of our and other RF-based localization approaches using WSNs and WLAN are summarized in Table 4. Our localization approach inflicts the lowest time costs of the compared schemes. Its installation includes only the mounting of the anchor nodes. The anchor network is formed and maintained autonomously. Due to the usage of LocMAC, the location node battery change interval is very long. The anchor nodes are mains-powered or equipped with large enough batteries requiring no or very infrequent maintenance.

The time costs of other range- and proximity-based approaches using WSNs are higher because the location and anchor node battery change intervals are significantly shorter than with our approach. In related WSN-based localization approaches location and anchor nodes are homogeneous at the communication level. Thus, anchor nodes are also considered to work with small batteries. The related proposals could also benefit from energy unconstrained anchor nodes, but since it is not considered in the papers, we will not consider it in this discussion either. The installation of other range- and proximity-based approaches using WSNs is easy for similar reasons than with our approach.

WLAN-based approaches incur bigger installation costs due to WLAN network planning procedure. These costs are hindered when existing network infrastructure can be exploited. Sufficient amount of APs need to cover every location in the localized area. Hence, also scenarios where a priorly installed WLAN network exists, may require fresh network planning. The anchor nodes are mains-powered and virtually maintenance-free. However, the high power consumption, and thus, short lifetime of the location nodes increases the maintenance costs.

The tedious training data sample database collection increases the installation costs of scene analysis approaches. Similar penalty is incurred regardless of the underlying network technology. In Table 4, the cost is modeled to be in the same order of a magnitude than WLAN localization network setup cost.

WSN node HW is comprised of simple components. Thus, the nodes can be very small and cheap. In our approach, the node unit costs are even smaller than with WSN-based solutions relying on RSSI. Some range and majority of proximity-based approaches can also cope without RSSI. However, the denser anchor network brings the aggregate anchor network space and capital costs closer to WLAN-based approaches.

In WLAN-based approaches, the location nodes need to be equipped with a relatively costly WLAN adapter. Often, a personal digital assistant (PDA) is used, but also simpler location nodes are possible. A WLAN AP incurs high space and capital costs compared to low-cost WSN nodes. WLAN requires that base stations are connected to a wired backbone (other infrastructure in Table 4). This increases the space and 
capital costs in sites with no a priori wired communication infrastructure.

\subsection{Location estimation}

The load of a location estimation algorithm can be divided into processing load and data memory load. The former is represented by the amount of operations needed for one location estimate. The latter is proportional to the amount of data storage needed by an algorithm. The load of most of the localization approaches is acceptable when the implementation is centralized and more powerful computers can be exploited. However, when decentralization is considered the location estimation algorithm load becomes a crucial factor.

The loads of different location estimation algorithms are summarized in Table 5. The processing load of our location estimation algorithm is very small. The bounding box resolution requires only simple comparison operations (subtraction) to find out the maximum and minimum values of coordinate sets. By using suitable data structures (e.g., a priority queue) even the amount of these simple operations can be minimized. Since the iterative bounding box algorithm is executed very infrequently, it is not considered here.

Our location estimation algorithm requires very small amount of data storage. For every anchor node used in one location estimation, only its coordinates and minimum received $\mathrm{LB}$ transmission power need to be temporarily stored. Furthermore, the transmission power to range mapping array needs to be saved. With four transmission power levels, the transmission power variable reserves only two bits and the size of the transmission power to range mapping array is four times two elements.

Traditional scene analysis incurs high computational load because a large sample database has to be processed. The JC [15] technique can reduce the needed amount of computation by orders of a magnitude by using clustering. For example, Youssef et al. report in [15] that while the JC technique requires on average about 40 operations per location estimate, RADAR requires approximately 460. MoteTrack [32] achieves similar benefits with sample database distribution to an WSN. Concurrently, scalability is enhanced. In scene analysis, the amount of memory needed to store the sample database is linearly proportional to the size of the covered area. Typically, the density of the training points dictate the maximum achievable location estimation accuracy [11]. Interpolation, used in $[11,14]$, can reduce the required density of the training points. Using interpolation, the required amount of training points still grows linearly as a function of the covered area, but the slope becomes smaller. The distributed approach of MoteTrack balances the data memory load to the anchor network.

Kalman filter is the most efficient implementation of Bayes filters in terms of computation and memory [40]. Still, considering embedded low-cost MCUs it requires a considerable amount of matrix multiplications to produce the posterior probability density of the location estimate. Memory is consumed by the state and measurement model, prior and posterior estimate, and covariance matrices. Using simple MCUs, whose processor does not include a hardware multiplier, the cost of multiplication is even further increased.

The highest processing load is inflicted by mathematical optimization algorithms. Typically, they are iterative and require matrix multiplications. For example in [37], the least squares implementation requires almost 2500 multiplication operations per one time step. In addition, with RSS-based solutions the solving of propagation model functions is needed to determine range estimates. The memory consumption is moderate and needed for the matrices involved in the computations.

Table 6 compares our and existing work with respect to localization accuracy. Since the presented experimental results in some references are somewhat scarce, the median accuracy values are used. When median accuracy is not given in the reference, average accuracy is used instead. Though median and average values describe different characteristics of the accuracy distribution, they can be used to indicate the scale of the results.

As can be seen from Table 6, our approach achieves accuracies in the same scale as related work. The submeter accuracy of Terwilliger et al. [26] can be mainly explained by the experimental setup, which includes only one room with high density of anchor nodes. The other approaches are experimented in multiroom office environments having multiple obstructions and realistic error sources.

The anchor node density of our approach is also in the same range as with related WSN-based approaches. For more in-depth comparison, the radio ranges of the devices used in the experiments should be known better. Localization performance with differing amount of anchor nodes would also give valuable additional information. In general, this kind of knowledge is not given in the related papers.

\section{DISCUSSION AND FUTURE WORK}

In this section, we discuss the minor changes needed to turn our approach decentralized and cooperative. Secondly, solutions for achieving even more low-power location nodes are described. The discussed issues, as well as anchor node energy-efficiency optimization, are part of our future work.

\subsection{Decentralization issues}

The design and implementation of the solution described thus far can be considered to be centralized, due to the gathering of the localization measurement data and the execution of the location resolver in a server. In the following, we will discuss the decentralization issues and show that our design can be transformed into a distributed one with minor changes.

The distributed flow of localization data exchanges is presented in Figure 21. In Figure 21(a), a location nodes transmits LBs to enable localization. The anchor nodes communicate heard LB data back to the location node. Also, the anchor node coordinates and local transmission power to range mapping values need to be sent. The three data units are aggregated into a localization data unit (LDU) frame which is transmitted to the location node as illustrated 
TABle 5: Location estimation load comparison.

\begin{tabular}{lclclcl}
\hline Algorithm & Our approach & $\begin{array}{l}\text { Traditional scene } \\
\text { analysis }\end{array}$ & JC technique [15] & $\begin{array}{l}\text { Distributed scene } \\
\text { analysis [32] }\end{array}$ & Bayesian filtering [40] $\begin{array}{l}\text { Mathematical } \\
\text { optimization }\end{array}$ \\
\hline $\begin{array}{l}\text { Processing load } \\
\text { Data memory load }\end{array}$ & Lowest & High & Medium & Medium & High & Highest \\
Hight & Highest & Highest & Balanced & Medium to high & Medium \\
\hline
\end{tabular}

TABle 6: Location estimation accuracy comparison. The anchor node density is expressed by $A / N$, where $A$ denotes size of the test area and $N$ denotes the anchor node amount used in the experiments.

\begin{tabular}{lll}
\hline Approach & $\begin{array}{l}\text { Median (M)/average } \\
\text { (A) accuracy [m] }\end{array}$ & $A / N[\mathrm{~m}]^{2}$ \\
\hline & WSN & \\
\hline Our approach (denser) & $1.70(\mathrm{M})$ & 30.43 \\
Our approach (sparser) & $3.30(\mathrm{M})$ & 58.33 \\
MoteTrack [32] & $2.00(\mathrm{M})$ & 87.10 \\
Shi et al. [29] & $2.60(\mathrm{M})$ & 20.00 \\
Alippi et al. [33] & $1.20(\mathrm{M})$ & 12.39 \\
Terwilliger et al. [26] & $0.60-0.70(\mathrm{~A})$ & $3.58-1.64$ \\
Online Person Tracking [30] & $2.00-3.80(\mathrm{M})$ & 84.00 \\
\hline & WLAN & 326.25 \\
\hline RADAR [12] & $2.94(\mathrm{M})$ & 607.20 \\
Elnahrawy et al. [14] & $1.80-6.00(\mathrm{M})$ & - \\
JC [15] & $1.20(\mathrm{M})$ & 72.00 \\
Kotanen et al. [9] & $2.60(\mathrm{~A})$ & - \\
Roos et al. [16] & $1.45(\mathrm{M})$ & \\
CMU-TMI [11] & $2.00(\mathrm{M})$ &
\end{tabular}

in Figure 21(b). This enables the location node to estimate its own location. If the location node is able to resolve a valid location, it continues normally by transmitting LBs in its next beacon cycle (back to Figure 21(a)). Optionally, it can publish its location by piggybacking it in the LBs. If the location node cannot resolve its location with the data extracted from the LDUs (i.e., the bounding boxes do not overlap), it runs the iterative bounding boxes algorithm. The new transmission power to range mapping values $\left(\operatorname{map}_{\mathrm{tx} 2 r}\right)$ are communicated to the anchor nodes by piggybacking them in transmitted LBs (Figure 21(c)). If the mapping values do not fit into a single packet, map $_{\mathrm{tx} 2 r}$ can be replaced with the corresponding path loss exponent value.

Thus, the distributed approach requires that

(1) the anchor nodes know their own location,

(2) the anchor network stores the local transmission power to range mapping values,

(3) the location nodes are able to estimate their own location, and

(4) the LDUs can be communicated to the location nodes.

For ad hoc operation, the first requirement would need an WSN self-localization approach, which is out of the scope of this discussion. Instead, we propose a simple, yet effective location initialization for a manually deployed anchor node network.

In the current solution, we position the anchor node icons manually to a floorplan map in a management UI. The UI communicates the locations to a server which stores them into a database. For making the anchor nodes locationaware, the location database could be disseminated to the anchor network after deployment.

The initial map $\operatorname{tx} 2 r$ values can be disseminated to the network similarly as anchor node locations. Location nodes piggyback corrected map $\operatorname{tx} 2 r$ values in LBs. Thus, the data in anchor nodes is continuously updated and the second requirement is satisfied.

The third requirement is enabled by the location resolver algorithm. It is designed to inflict low-processing overhead and resource consumption. Thus, it can be executed in the resource-constrained location nodes.

In order to reliably communicate LDU data to the location node (requirement four), the downlink phase of LocMAC base needs some changes. The distributed operation requires that multiple anchor nodes can communicate LDU data to the location node inside one beacon cycle. The current LocMAC base downlink includes only one slot. Thus, only one anchor node can send data to the location node in a beacon cycle, which is infeasible in the distributed scenario.

Next, we outline an example solution for more effective downlink communication. The current downlink slot is replaced with a longer random access period. This will increase the location node power consumption to some extent depending on the duration of the period. To enable the communication of most valuable range estimates while keeping the random access period as short as possible, a priority-based approach similar to the one represented in [75] is used.

The anchor nodes are assigned priorities according to the reliability of their distance estimates. The higherpriority anchor nodes have higher probability to access the medium. RF path loss can be modeled to follow log-normal distribution, and thus, RF path loss -based ranging gives larger absolute errors as range increases [24]. Hence, distance estimates to anchor nodes that are closer are also more reliable and the anchor nodes receiving LBs with smaller transmission power are assigned higher priority level.

\subsection{Further energy optimizations}

If lower location refresh rate is tolerated by the application, LocMAC performance can be tuned to achieve even longer location node lifetimes. First, as indicated 
earlier, the location node beacon cycle can be extended. Secondly, transmissions can be minimized by circulating LB transmission powers. For example using two LBs, the first LB could be sent with circulated transmission powers and the second always with highest transmission power. This would result in robust coarse-grained location estimation, since the maximum transmission range is used every beacon cycle. Furthermore, more fine grained location estimation with lower refresh rate would be achieved with the circulated LBs.

If instead of continuous tracking an alarm-type application is utilized, the location nodes can be in low-power state most of the time. Upon an event, for example, a button push by the person carrying the location node, the location node could start running LocMAC until the event was acknowledged. This would result in extended location node lifetime and user privacy, while achieving localization when required.

\section{CONCLUSIONS}

In this paper, we presented the design, implementation, and evaluation of a localization approach using minimal hardware. The design followed six objectives: location node energy-efficiency, scalability, bidirectional communication between location nodes and anchor nodes, localization with low-cost hardware, room-level accuracy, and real-time operation.

The nodes were based on a novel MAC, called LocMAC, designed for localization purposes. We showed that LocMAC outperforms current state-of-the-art WSN MAC protocols in location node energy-efficiency.

A fully calibration-free location resolver algorithm capable of location estimation with coarse-grained and unreliable ranging measurements was presented. The localization showed point-based accuracies in the range of $1 \mathrm{~m}$ to $7 \mathrm{~m}$, and the room was determined correctly $89.7 \%$ of the cases when every room in the test area was equipped with an anchor node. Halving the anchor node amount hindered the results but showed resilience against anchor node failure and incomplete data.

In this paper, we have demonstrated that continuous localization is possible with nodes having minimal hardware and reaching lifetime of several years with small batteries.

\section{REFERENCES}

[1] N. Bulusu, J. Heidemann, and D. Estrin, "GPS-less low-cost outdoor localization for very small devices," IEEE Personal Communications, vol. 7, no. 5, pp. 28-34, 2000.

[2] B. Schilit, N. Adams, and R. Want, "Context-aware computing applications," in Proceedings of IEEE Workshop on Mobile Computing Systems and Applications (WMCSA '94), pp. 85-90, Santa Cruz, Calif, USA, December 1994.

[3] J. Syrjärinne, Studies of modern techniques for personal positioning, Ph.D. dissertation, Tampere University of Technology, Tampere, Finland, March 2001.

[4] I. Stojmenovic, "Position-based routing in ad hoc networks," IEEE Communications Magazine, vol. 40, no. 7, pp. 128-134, 2002.
[5] H. Frey and D. Görgen, "Geographical cluster-based routing in sensing-covered networks," IEEE Transactions on Parallel and Distributed Systems, vol. 17, no. 9, pp. 899-911, 2006.

[6] H. Karl and A. Willig, Eds., Protocols and Architectures for Wireless Sensor Networks, John Wiley \& Sons, New York, NY, USA, 2005.

[7] P. Misra and P. Enge, Global Positioning System: Signals, Measurements, and Performance, Ganga-Jumuna Press, Lincoln, Mass, USA, 2001.

[8] T. Kitasuka, T. Nakanishi, and A. Fukuda, "Design of WIPS: WLAN-based indoor positioning system," Korea Multimedia Society, vol. 7, no. 4, pp. 15-29, 2003.

[9] A. Kotanen, M. Hännikäinen, H. Leppäkoski, and T. D. Hämäläinen, "Positioning with IEEE 802.11b wireless LAN," in Proceedings of the 14th IEEE Iternational Symposium on Personal, Indoor and Mobile Radio Communications (PIMRC '03), vol. 3, pp. 2218-2222, Bejing, China, September 2003.

[10] T. D. Hodes, R. H. Katz, E. Servan-Schreiber, and L. Rowe, "Composable ad-hoc mobile services for universal interaction," in Proceedings of the 3rd Annual International Conference on Mobile Computing and Networking (MOBICOM '97), pp. 1-12, Budapest, Hungary, September 1997.

[11] A. Smailagic and D. Kogan, "Location sensing and privacy in a context-aware computing environment," IEEE Wireless Communications, vol. 9, no. 5, pp. 10-17, 2002.

[12] P. Bahl and V. N. Padmanabhan, "RADAR: an in-building RF-based user location and tracking system," in Proceedings of the 19th Annual Joint Conference of IEEE Computer and Communications Societies (INFOCOM '00), vol. 2, pp. 775784, Tel Aviv, Israel, March 2000.

[13] E. Elnahrawy, X. Li, and R. P. Martin, "Using area-based presentations and metrics for localization systems in wireless LANs," in Proceedings of the 29th Annual IEEE International Conference on Local Computer Networks (LCN '04), pp. 650657, Tampa, Fla, USA, November 2004.

[14] E. Elnahrawy, X. Li, and R. P. Martin, "The limits of localization using signal strength: a comparative study," in Proceedings of the 1st Annual IEEE Communications Society Conference on Sensor and Ad Hoc Communications and Networks (SECON '04), pp. 406-414, Santa Clara, Calif, USA, October 2004.

[15] M. A. Youssef, A. Agrawala, and A. U. Shankar, "WLAN location determination via clustering and probability distributions," in Proceedings of the 1st IEEE International Conference on Pervasive Computing and Communications (PerCom '03), pp. 143-150, Dallas, Tex, USA, September 2003.

[16] T. Roos, P. Myllymäki, H. Tirri, P. Misikangas, and J. Sievänen, "A probabilistic approach to WLAN user location estimation," International Journal of Wireless Information Networks, vol. 9, no. 3, pp. 155-164, 2002.

[17] I. F. Akyildiz, W. Su, Y. Sankarasubramaniam, and E. Cayirci, "Wireless sensor networks: a survey," Computer Networks, vol. 38, no. 4, pp. 393-422, 2002.

[18] D. Culler, D. Estrin, and M. Srivastava, "Guest editors' introduction: overview of sensor networks," Computer, vol. 37, no. 8, pp. 41-49, 2004.

[19] I. F. Akyildiz, W. Su, Y. Sankarasubramaniam, and E. Cayirci, "A survey on sensor networks," IEEE Communications Magazine, vol. 40, no. 8, pp. 102-114, 2002.

[20] M. Kuorilehto, J. Suhonen, M. Kohvakka, M. Hännikäinen, and T. D. Hämäläinen, "Experimenting TCP/IP for low-power wireless sensor networks," in Proceedings of the 17th IEEE International Symposium on Personal, Indoor and Mobile Radio 
Communications (PIMRC '06), pp. 1-6, Helsinki, Finland, September 2006.

[21] M. Kohvakka, J. Suhonen, M. Kuorilehto, V. A. Kaseva, M. Hännikäinen, and T. D. Hämäläinen, "Energy-efficient neighbor discovery protocol for mobile wireless sensor networks," Ad Hoc Networks. November 14, 2006, Article in Press.

[22] A. El-Hoiydi and J.-D. Decotignie, "Low power downlink MAC protocols for infrastructure wireless sensor networks," Mobile Networks and Applications, vol. 10, no. 5, pp. 675-690, 2005.

[23] J. Hightower and G. Borriello, "Location systems for ubiquitous computing," Computer, vol. 34, no. 8, pp. 57-66, 2001.

[24] N. Patwari, J. N. Ash, S. Kyperountas, A. O. Hero III, R. L. Moses, and N. S. Correal, "Locating the nodes: cooperative localization in wireless sensor networks," IEEE Signal Processing Magazine, vol. 22, no. 4, pp. 54-69, 2005.

[25] C. Wang and X. Li, "Sensor localization under limited measurement capabilities," IEEE Network, vol. 21, no. 3, pp. 16-23, 2007.

[26] M. Terwilliger, A. Gupta, V. Bhuse, Z. Kamal, and M. Salahuddin, "A localization system using wireless network sensors: a comparison of two techniques," in Proceedings of the 1st Workshop on Positioning, Navigation and Communication (WPNC '04), Hanover, Germany, March 2004.

[27] G. S. Paschos, E. D. Vagenas, and S. A. Kotsopoulos, "Realtime localization for wireless sensor networks with multiple beacon transmissions," in Proceedings of the 5th International Network Conference (INC '05), Island of Samos, Greece, July 2005.

[28] J. Hightower, R. Want, and G. Borriello, "SpotON: an indoor 3D location sensing technology based on RF signal strength," Tech. Rep. UW CSE 00-02-02, Department of Computer Science and Engineering, University of Washington, Seattle, Wash, USA, February 2000, http://portolano.cs.washington.edu/projects/spoton/.

[29] W. Shi, C. Huang, M. Shao, Y. Cheng, and Z. Chen, "Indoor localization scheme in wireless sensor networks using spatial information," in Proceedings of the International Conference on Wireless Communications, Networking and Mobile Computing (WiCOM '06), pp. 1-5, Wuhan, China, September 2006.

[30] X. An, J. Wang, R. V. Prasad, and I. G. M. M. Niemegeers, "OPT: online person tracking system for context-awareness in wireless personal network (demo)," in Proceedings of 2nd International Workshop on Multi-hop Ad Hoc Networks: From Theory to Reality (REALMAN '06), pp. 47-54, Florence, Italy, May 2006.

[31] T. He, C. Huang, B. M. Blum, J. A. Stankovic, and T. Abdelzaher, "Range-free localization schemes for large scale sensor networks," in Proceedings of the 9th Annual International Conference on Mobile Computing and Networking (MOBICOM '03), pp. 81-95, San Diego, Calif, USA, September 2003.

[32] K. Lorincz and M. Welsh, "MoteTrack: a robust, decentralized approach to RF-based location tracking," in Proceedings of the 1st International Workshop on Location- and ContextAwareness (LoCA '05), vol. 3479, pp. 63-82, Oberpfaffenhofen, Germany, May 2005.

[33] C. Alippi, A. Mottarella, and G. Vanini, "A RF map-based localization algorithm for indoor environments," in Proceedings of IEEE International Symposium on Circuits and Systems (ISCAS '05), vol. 1, pp. 652-655, Kobe, Japan, May 2005.

[34] A. Ward, A. Jones, and A. Hopper, "A new location technique for the active office," IEEE Personal Communications, vol. 4, no. 5, pp. 42-47, 1997.
[35] N. B. Priyantha, A. Chakraborty, and H. Balakrishnan, "The cricket location-support system," in Proceedings of the 6th Annual International Conference on Mobile Computing and Networking (MOBICOM '00), pp. 32-43, Boston, Mass, USA, August 2000.

[36] N. B. Priyantha, A. K. L. Miu, H. Balakrishnan, and S. Teller, "The cricket compass for context-aware mobile applications," in Proceedings of the 7th Annual International Conference on Mobile Computing and Networking, (MOBICOM '01), pp. 114, Rome, Italy, July 2001.

[37] A. Smith, H. Balakrishnan, M. Goraczko, and N. Priyantha, "Tracking moving devices with the cricket location system," in Proceedings of the 2nd International Conference on Mobile Systems, Applications and Services (MobiSys '04), pp. 190-202, Boston, Mass, USA, June 2004.

[38] R. Want, A. Hopper, V. Falcao, and J. Gibbons, "The active badge location system," ACM Transactions on Information Systems, vol. 10, no. 1, pp. 91-102, 1992.

[39] A. Harter and A. Hopper, "A distributed location system for the active office," IEEE Network, vol. 8, no. 1, pp. 62-70, 1994.

[40] D. Fox, J. Hightower, L. Liao, D. Schulz, and G. Borriello, "Bayesian filtering for location estimation," IEEE Pervasive Computing, vol. 2, no. 3, pp. 24-33, 2003.

[41] J. Hightower, B. Brumitt, and G. Borriello, "The location stack: a layered model for location in ubiquitous computing," in Proceedings of the 4th IEEE Workshop on Mobile Computing Systems and Applications (WMCSA '02), pp. 22-28, Callicoon, NY, USA, June 2002.

[42] M. Kohvakka, J. Suhonen, M. Hännikäinen, and T. D. Hämäläinen, "Transmission power based path loss metering for wireless sensor networks," in Proceedings of the 17th IEEE International Symposium on Personal, Indoor and Mobile Radio Communications (PIMRC '06), pp. 1-5, Helsinki, Finland, September 2006.

[43] A. El-Hoiydi and J.-D. Decotignie, "WiseMAC: an ultra low power MAC protocol for multi-hop wireless sensor networks," in Proceedings of the 1st International Workshop on Algorithmic Aspects of Wireless Sensor Networks (ALGOSENSORS '04), vol. 3121, pp. 18-31, Turku, Finland, July 2004.

[44] J. Polastre, J. Hill, and D. Culler, "Versatile low power media access for wireless sensor networks," in Proceedings of the 2nd International Conference on Embedded Networked Sensor Systems (SenSys '04), pp. 95-107, Baltimore, Md, USA, November 2004.

[45] K.-J. Wong and D. K. Arvind, "SpeckMAC: low-power decentralised MAC protocols for low data rate transmissions in specknets," in Proceedings of the 2nd International Workshop on Multi-hop Ad Hoc Networks: From Theory to Reality (REALMAN '06), pp. 71-78, Florence, Italy, May 2006.

[46] M. Buettner, G. V. Yee, E. Anderson, and R. Han, "X-MAC: a short preamble MAC protocol for duty-cycled wireless sensor networks," in Proceedings of the 4th International Conference on Embedded Networked Sensor Systems (SenSys '06), pp. 307320, Boulder, Colo, USA, October-November 2006.

[47] W. Ye, F. Silva, and J. Heidemann, "Ultra-low duty cycle MAC with scheduled channel polling," in Proceedings of the 4th International Conference on Embedded Networked Sensor Systems (SenSys '06), pp. 321-334, Boulder, Colo, USA, October-November 2006.

[48] A. El-Hoiydi, "Spatial TDMA and CSMA with preamble sampling for low power ad hoc wireless sensor networks," in Proceedings of the 7th International Symposium on Computers and Communications (ISCC '02), pp. 685-692, Taormina, Italy, July 2002. 
[49] W. Ye, J. Heidemann, and D. Estrin, "Medium access control with coordinated adaptive sleeping for wireless sensor networks," IEEE Transactions on Networking, vol. 12, no. 3, pp. 493-506, 2004.

[50] T. van Dam and K. Langendoen, "An adaptive energy-efficient MAC protocol for wireless sensor networks," in Proceedings of the 1st International Conference on Embedded Networked Sensor Systems (SenSys '03), pp. 171-180, Los Angeles, Calif, USA, November 2003.

[51] IEEE Std. 802.15.4, "IEEE standard for information technology-telecommunications and information exchange between systems-local and metropolitan area networksspecific requirements-part 15.4: wireless medium access control (MAC) and physical layer (PHY) specifications for low-rate wireless personal area networks (LR-WPAN)," 2003.

[52] K. Sohrabi, J. Gao, V. Ailawadhi, and G. J. Pottie, "Protocols for self-organization of a wireless sensor network," IEEE Personal Communications, vol. 7, no. 5, pp. 16-27, 2000.

[53] W. B. Heinzelman, A. P. Chandrakasan, and H. Balakrishnan, "An application-specific protocol architecture for wireless microsensor networks," IEEE Transactions on Wireless Communications, vol. 1, no. 4, pp. 660-670, 2002.

[54] G. Pei and C. Chien, "Low power TDMA in large wireless sensor networks," in Proceedings of IEEE Military Communications Conference, IEEE Communications for Network-Centric Operations: Creating the Information Force (MILCOM '01), vol. 1, pp. 347-351, McLean, Va, USA, October 2001.

[55] V. Rajendran, K. Obraczka, and J. J. Garcia-Luna-Aceves, "Energy-efficient, collision-free medium access control for wireless sensor networks," in Proceedings of the 1st International Conference on Embedded Networked Sensor Systems (SenSys '03), pp. 181-192, Los Angeles, Calif, USA, November 2003.

[56] T. Wu and S. Biswas, "A self-reorganizing slot allocation protocol for multi-cluster sensor networks," in Proceedings of the 4th International Symposium on Information Processing in Sensor Networks (IPSN '05), pp. 309-316, Los Angeles, Calif, USA, April 2005.

[57] M. Kohvakka, M. Hännikäinen, and T. D. Hämäläinen, "Ultra low energy wireless temperature sensor network implementation," in Proceedings of the 16th IEEE International Symposium on Personal, Indoor and Mobile Radio Communications (PIMRC '05), vol. 2, pp. 801-805, Berlin, Germany, September 2005.

[58] M. Kohvakka, M. Hännikäinen, and T. D. Hämäläinen, "Energy optimized beacon transmission rate in a wireless sensor network," in Proceedings of the 16th IEEE International Symposium on Personal, Indoor and Mobile Radio Communications (PIMRC '05), vol. 2, pp. 1269-1273, Berlin, Germany, September 2005.

[59] C. Intanagonwiwat, R. Govindan, and D. Estrin, "Directed diffusion: a scalable and robust communication paradigm for sensor networks," in Proceedings of the 6th Annual International Conference on Mobile Computing and Networking (MOBICOM '00), pp. 56-67, Boston, Mass, USA, August 2000.

[60] H. S. Kim, T. F. Abdelzaher, and W. H. Kwon, "Minimumenergy asynchronous dissemination to mobile sinks in wireless sensor networks," in Proceedings of the 1st International Conference on Embedded Networked Sensor Systems (SenSys '03), pp. 193-204, Los Angeles, Calif, USA, November 2003.

[61] H. Pham and S. Jha, "Addressing mobility in wireless sensor media access protocol," in Proceedings of the Intelligent Sensors, Sensor Networks and Information Processing Conference (ISSNIP '04), pp. 113-118, Melbourne, Australia, December 2004.
[62] M. Ali, T. Suleman, and Z. A. Uzmi, "MMAC: a mobilityadaptive, collision-free MAC protocol for wireless sensor networks," in Proceedings of the 24th IEEE International Performance, Computing, and Communications Conference (IPCCC '05), pp. 401-407, Phoenix, Ariz, USA, April 2005.

[63] P. Raviraj, H. Sharif, M. Hempel, S. Ci, H. H. Ali, and J. Youn, "A mobility based link layer approach for mobile wireless sensor networks," in Proceedings of IEEE International Conference on Electro Information Technology (EIT '05), p. 6, Lincoln, Neb, USA, May 2005.

[64] A. Koubâa, M. Alves, and E. Tovar, "Lower protocol layers for wireless sensor networks: a survey," Tech. Rep. HURRAYTR-051101, Polytechnic Institute of Porto, Porto, Portugal, Novenber 2005.

[65] IEEE 802.11 working group, http://grouper.ieee.org/groups/ $802 / 11 /$.

[66] J. Elson, L. Girod, and D. Estrin, "Fine-grained network time synchronization using reference broadcasts," ACM SIGOPS Operating Systems Review, vol. 36, no. SI, pp. 147-163, 2002.

[67] C.-K. Toh, V. Vassiliou, G. Guichal, and C.-H. Shih, "MARCH: a medium access control protocol for multihop wireless ad hoc networks," in Proceedings of the 21st Century Military Communications Conference (MILCOM '00), vol. 1, pp. 512516, Los Angeles, Calif, USA, October 2000.

[68] L. G. Roberts, "Aloha packet system with and without slots and capture," ACM SIGCOMM Computer Communication Review, vol. 5, no. 2, pp. 28-42, 1975.

[69] K. Langendoen and N. Reijers, "Distributed localization in wireless sensor networks: a quantitative comparison," Computer Networks, vol. 43, no. 4, pp. 499-518, 2003.

[70] A. Savvides, H. Park, and M. B. Srivastava, "The $n$-hop multilateration primitive for node localization problems," Mobile Networks and Applications, vol. 8, no. 4, pp. 443-451, 2003.

[71] T. Parker and K. Langendoen, "Refined statistic-based localisation for ad-hoc sensor networks," in Proceedings of IEEE Global Telecommunications Conference (GlobeCom '04), pp. 90-95, Dallas, Tex, USA, November-December 2004.

[72] “Texas instruments," December 2007, http://www.ti.com/.

[73] "Nordic semiconductor," July 2007, http://www.nordicsemi .no/.

[74] F. Jin, H.-A. Choi, and S. Subramaniam, "Hardware-aware communication protocols in low energy wireless sensor networks," in Proceedings of IEEE Military Communications Conference (MILCOM '03), vol. 1, pp. 676-681, Monterey, Calif, USA, October 2003.

[75] D. Wang, C. Comaniciu, and U. Tureli, "Cross layer design for localization and MAC," in Proceedings of the 40th Annual IEEE Conference on Information Sciences and Systems (CISS '06), pp. 407-412, Princeton, NJ, USA, March 2006. 